\title{
BOOTSTRAPPING INDUCTIVE AND COINDUCTIVE TYPES IN HASCASL
}

\author{
LUTZ SCHRÖDER
}

DFKI Bremen and Department of Computer Science, University of Bremen, Germany e-mail address: Lutz.Schroeder@dfki.de

\begin{abstract}
We discuss the treatment of initial datatypes and final process types in the wide-spectrum language HasCASL. In particular, we present specifications that illustrate how datatypes and process types arise as bootstrapped concepts using HasCASL's type class mechanism, and we describe constructions of types of finite and infinite trees that establish the conservativity of datatype and process type declarations adhering to certain reasonable formats. The latter amounts to modifying known constructions from HOL to avoid unique choice; in categorical terminology, this means that we establish that quasitoposes with an internal natural numbers object support initial algebras and final coalgebras for a range of polynomial functors, thereby partially generalising corresponding results from topos theory. Moreover, we present similar constructions in categories of internal complete partial orders in quasitoposes.
\end{abstract}

\section{INTRODUCTION}

The formally stringent development of software in a unified process calls for wide-spectrum languages that support all stages of the formal development process, including abstract requirements, design, and implementation. In the CASL language family [3], this role is played by the higher-order CASL extension HASCASL [29, 28]. Like in first-order CASL, a key feature of HASCASL is support for inductive datatypes, which appear in the specification of the functional correctness of software. In the algebraic-coalgebraic language CoCASL [13], this concept is complemented by coinductive types, which appear as state spaces of reactive processes. Many issues revolving around types of either kind gain in complexity in the context of the enriched language HASCASL; this is related both to the presence of additional language features such as higher order types and type class polymorphism and to the nature of the underlying logic of HASCASL, an intuitionistic higher order logic of partial functions without unique choice which may, with a certain margin of error, be thought of as the internal logic of quasitoposes (more precisely, it is the internal logic of partial cartesian closed categories with equality [25, 26]).

1998 ACM Subject Classification: D.2.1, E.1, F.3.1, F.3.2, F.4.1.

Key words and phrases: datatypes, process types, software specification, quasitoposes, partial $\lambda$-calculus. This work is an extended version of [27.

Work performed under DFG-project HASCASL (KR 1191/7-2) and BMBF-project FormalSafe (FKZ 01IW07002).

DOI:10.2168/LMCS-4 (4:17) 2008

(C) L. Schröder

(c) Creative Commons 
Here, we discuss several aspects of HASCASL's concept of inductive datatype, as well as the perspective of adding coinductive types to HASCASL. To begin, we present the syntax and semantics of inductive datatypes, which may be equipped with reachability constraints or initiality constraints; both types of constraints may be relatively involved due to the fact that constructor arguments may have complex composite types. We then go on to show how initial datatypes may be specified in terms of HASCASL's type class mechanism. On the one hand, this shows that initial datatypes need not be regarded as a built-in language feature, but may be considered as belonging into a 'HASCASL prelude'. On the other hand, the specifications in question give a good illustration of how far the type class mechanism may be stretched. We then briefly discuss how a simple dualisation of these specifications describes final process types in the style of CoCASL; thus, the introduction of such types into HASCASL would merely constitute additional syntactic sugar (although concerning the relationship to CASL and COCASL, for both datatypes and process types certain caveats apply related to HASCASL's Henkin semantics).

Finally, we tackle the issue of the conservativity of datatype and process type declarations. We follow the method employed in standard HOL [18, 2], which consists in defining a universal type of trees and then carving out the desired inductive or coinductive types. However, the constructions need to be carefully adapted in order to cope with the lack of unique choice. Abstracting our results to the categorical level, we prove, in partial generalisation of corresponding results for toposes [16, 10, that any quasitopos (indeed, any partial cartesian closed category with equality and finite coproducts) with nno supports initial algebras and final coalgebras for certain classes of polynomial functors. Moreover, we obtain corresponding results for datatypes and process types equipped with complete partial orders (the former are called free domains in HASCASL). These types serve as the correspondent of programming language datatypes in HASCASL's internal modelling of denotational semantics.

The material is organised as follows. We recall some aspects of the syntax and semantics of HASCASL, including the relationship between HASCASL' Henkin models and categorical models, in Sect. 1. In Sect. 2, we expand on the semantics of generated and free datatypes in HASCASL. These two sections summarise material from [26, 28]. We then go on to

present the bootstrapped specification of the syntax and semantics of signature functors and inductive datatypes using the type class mechanism in Sect. 3. In Sect. 4 we discuss how these concepts extend naturally to coinductive process types. We present the constructions establishing the conservativity of datatype and process type declarations in Sect. 5. Finally, we recall the modelling of general recursive programs by means of an adapted version of domain theory in Sect. 6, and show how the constructions of plain initial datatypes and final process types can be modified to obtain corresponding constructions on domains.

\section{HASCASL}

The wide-spectrum language HASCASL [29] extends the standard algebraic specification language CASL by intuitionistic partial higher order logic, equipped with a set-theoretic Henkin semantics, an extensive type class mechanism, and HOLCF-style support for recursive programming. HASCASL moreover provides support for functional-imperative specification and programming in the shape of monad-based computational logics [30, 32, 31, 35]. Tool support for HASCASL is provided in the framework of the Bremen heterogeneous tool set Hets [12]. We expect the reader to be familiar with the basic CASL syntax (whose use 
in our examples is, at any rate, largely self-explanatory), referring to [3, 14] for a detailed language description. Below, we review the HASCASL language features most relevant for the understanding of the present work, namely type class polymorphism and certain details of HASCASL's higher order logic; see 28, for a full language definition. Moreover, we recall HASCASL's Henkin semantics and its relation to categorical models in quasitoposes and, more generally, partial cartesian closed categories with equality [26].

1.1. The internal logic of HasCasl. The logic of HASCASL is based on the partial $\lambda$-calculus [11. It is distinguished from standard HOL by having intuitionistic truth values and partial function types $t \rightarrow ? s$ (besides total function types $t \rightarrow s$ ); $\lambda$-abstractions $\lambda x: t \bullet \alpha$ denote partial functions, i.e. inhabitants of partial function types $t \rightarrow ? s$, while total $\lambda$-abstractions, inhabiting the total function type $t \rightarrow s$, are denoted $\lambda x: s \bullet ! \alpha$. There are moreover a unit type Unit, with unique inhabitant (), and product types $s \times t$. Predicates then arise as partial functions into Unit, where definedness is understood as satisfaction, and the type of truth values is Logical $=$ Unit $\rightarrow$ ? Unit. We denote application of a function $f$ to an argument $x$ as $f x$, under the convention that application is left-associative. As in [26], we moreover denote by $\alpha \uparrow \phi$ the restriction of a term $\alpha$ to a formula $\phi$, i.e. $\alpha \uparrow \phi$ is defined iff $\alpha$ is defined and $\phi$ holds, and in this case is equal to $\alpha$ (essentially, $\uparrow$ is just the first projection).

In a partial setting, there are numerous readings of equality; here, we require strong equality, denoted $=$ and read 'one side is defined iff the other is, and in this case, both sides are equal', as well as existential equality, denoted $\stackrel{e}{=}$ and read 'both sides are defined and equal'. Equality of terms in the partial $\lambda$-calculus is axiomatised largely as expected [11, 26], with a few subtleties attached to partiality - e.g. $\beta$-equality $(\lambda x: t \bullet \alpha) \gamma=\alpha[\gamma / x]$ holds only if the term $\gamma$ is defined. We assume that equality is internal, i.e. that there exists on every type a binary predicate representing existential equality, also written $\stackrel{e}{=}$; this defines the partial $\lambda$-calculus with equality. In the partial $\lambda$-calculus with equality, an intuitionistic predicate logic is defined in the standard way (see e.g. [25, 26]) by abbreviations such as

$$
\forall x: t \bullet \phi=((\lambda x: t \bullet \phi) \stackrel{e}{=}(\lambda x: a \bullet())),
$$

where () is the unique inhabitant of Unit. The arising logic includes higher-order universal and existential quantifiers $\forall, \exists$, propositional connectives $\wedge, \vee, \Rightarrow, \Leftrightarrow, \neg$, and truth values $\top, \perp$.

The difference between the HASCASL logic and the more familiar topos logic [9] is the absence of unique choice [26], where we say that a type a admits unique choice if $a$ supports unique description terms of the form $(\iota x: a . \phi): a$ designating the unique element $x$ of $a$ satisfying the formula $\phi$ (which may of course mention $x$ ), if such an element exists uniquely (this is like Isabelle/HOL's THE [15]). In HASCASL, the unique choice principle may be imposed if desired by means of a polymorphic axiom [28]. The lack of unique choice requires additional effort in the construction of tree types establishing the conservativity of datatype and process type declarations; this is the main theme of Sect. 5. The motivation justifying this effort is twofold:

- Making do without unique choice essentially amounts to admitting models in quasitoposes rather than just in toposes (see Section 1.3). Interesting set-based quasitoposes include pseudotopological spaces and reflexive relations; further typical examples are categories of extensional presheaves, including e.g. the category of reflexive logical relations, and categories of assemblies, both appearing in the context of realisability models [19, 23]. In 
particular, the category of $\omega$-sets is a quasitopos but not a topos; it is embedded as a full subcategory into the effective topos, whose objects however have a much more involved description than $\omega$-sets [19]. Quasitoposes also play a role in the semantics of parametric polymorphism [4].

- A discipline of avoiding unique choice leads to constructions which may be easier to handle in machine proofs than ones containing unique description operators; cf. e.g. the explicit warning from [15], Sec. 5.10:

"Description operators can be hard to reason about. Novices should try to avoid them. Fortunately, descriptions are seldom required."

As we shall discuss below, one occasion where the theory development of Isabelle/HOL does require description operators is the construction of datatypes, and our results shed some light on the question to which extend this can be avoided.

1.2. Type class polymorphism. HASCASL's shallow polymorphism revolves around a notion of type class. Type classes are syntactic subsets of kinds, where kinds are formed from classes, including a base class Type of all types, and the type function arrow $\rightarrow$. Classes are declared by means of the keyword class; e.g.

$$
\text { class Functor }<\text { Type } \rightarrow \text { Type }
$$

declares a class Functor of type constructors, i.e. operations taking types to types. Types are declared with associated classes (or with default class Type) by means of the keyword type; e.g. a type constructor $F$ of class Functor is declared by writing

type $F$ : Functor

Such declarations may be generic; e.g. if $\operatorname{Ord}$ is a class, then we may write

$$
\begin{array}{ll}
\text { var } a, b: \text { Ord } \\
\text { type } a \times b: \text { Ord }
\end{array}
$$

thus imposing that the class Ord is closed under products; note how the keyword var is used for both standard variables and type variables. Operations and axioms may be polymorphic over any class, i.e. types of operations and variables may contain type variables with assigned classes.

In order to ensure the institutional satisfaction condition (invariance of satisfaction under change of notation), polymorphism is equipped with an extension semantics [33]; the only point to note for purposes of this work is that as a consequence, a specification extension is, in CASL terminology, (model-theoretically) conservative, i.e. admits expansions of models, iff it only introduces names for entities already expressible in the present signature. In the case of types, this means that e.g. a datatype declaration is conservative iff it can be implemented as a subtype of an existing type. 
1.3. Henkin Models and Partial Cartesian Closed Categories. The set-theoretic semantics of HASCASL is given by intensional Henkin models, where function types are equipped with application operators but are neither expected to contain all set-theoretic functions nor indeed to consist of functions; in particular, different elements of the function type may induce the same set-theoretic function. Such models are essentially equivalent to models in (varying!) partial cartesian closed categories (pccc's) with equality [26]; these categories are slightly more general than quasitoposes [1], which can be seen as finitely cocomplete pccc's with equality. Below, we summarise some of the details of the categorical viewpoint; we refer to [26, 28] for the full definition of intensional Henkin models.

A dominion [22] on a category $\mathbf{C}$ is a class $\mathcal{M}$ of monomorphisms in $\mathbf{C}$ which contains all identities and is closed under composition and pullback stable, the latter in the sense that pullbacks, or inverse images, of $\mathcal{M}$-morphisms along arbitrary morphisms exist and are in $\mathcal{M}$. The pair $(\mathbf{C}, \mathcal{M})$ is called a dominional category. A partial morphism $X \rightarrow Y$ in $(\mathbf{C}, \mathcal{M})$ is a span $X \stackrel{m}{\longleftrightarrow} D \stackrel{f}{\longrightarrow} Y$, where $m \in \mathcal{M}$, taken modulo isomorphic change of $D$. Partial morphisms $(m, f)$ are composed by pullback formation. Intuitively, $(m, f)$ is a partial map defined on the subobject $D$ of $X$. The partial morphisms in $(\mathbf{C}, \mathcal{M})$ form a category $\mathbf{P}(\mathbf{C}, \mathcal{M})$ (with small hom-sets if $\mathbf{C}$ is $\mathcal{M}$-wellpowered [1]), into which $\mathbf{C}$ is embedded by mapping a morphism $f$ to the partial morphism $(i d, f)$. If $\mathbf{C}$ is cartesian, i.e. has a terminal object 1 and binary products $A \times B$, then $\mathbf{C}$ is a partial cartesian closed category (pccc) if the functor

$$
\mathbf{C} \stackrel{-\times A}{\longrightarrow} \mathbf{C} \hookrightarrow \mathbf{P}(\mathbf{C}, \mathcal{M})
$$

has a right adjoint for each object $A$ in $\mathbf{C}$. If in addition, $\mathcal{M}$ contains all diagonal morphisms $A \rightarrow A \times A$, then a monomorphism $f$ in $\mathbf{C}$ is extremal iff $f$ is regular iff $f \in \mathcal{M}$ (see e.g. [1] for definitions of extremal and regular monomorphisms). In this case, $\mathbf{C}$ is called a pccc with equality.

Remark 1.1. The above constructive approach to partial maps is complemented by a variety of direct approaches which take the category of partial maps as basic and axiomatise its properties. The details of these approaches and their relationship to the constructive approach are discussed in some breadth in [26].

It has been shown in [26] that one has an equivalence between theories in the partial $\lambda$-calculus with equality and pccc's with equality. Here, a theory consists of a set of basic types, from which composite types are obtained inductively by forming partial function types $s_{1} \times \cdots \times s_{n} \rightarrow$ ? $t$, a set of basic operations with assigned types, and a set of axioms, expressed as existentially conditioned equations (ece's) in this signature. Here, an existentially conditioned equation is a sentence of the form $\bigwedge_{i=1}^{n}$ def $\alpha_{i} \Rightarrow \beta \stackrel{e}{=} \gamma$, where $\beta, \gamma$, and the $\alpha_{i}$ are terms formed from the basic operations, application, $\lambda$-abstraction, and typed variables from a given context, and $\operatorname{def} \alpha$ abbreviates the formula $\alpha \stackrel{e}{=} \alpha$, which states that the term $\alpha$ is defined. Note that since the higher-order internal logic recalled in Section 1.1 is defined through equality, a theory may alternatively be seen as having axioms using the full power of the internal logic.

In the correspondence between categories and theories, one associates to every pccc with equality, $\mathbf{C}$, an internal language $\operatorname{Th}(\mathbf{C})$ which has the objects of $\mathbf{C}$ as basic types and the partial morphisms as operations, as well as all ece's expressed in this language which hold in $\mathbf{C}$ as axioms. Conversely, one associates to every theory $\mathcal{T}$ in the partial $\lambda$-calculus with equality a pccc with equality, $\mathrm{Cl}(\mathcal{T})$, the classifying category of $\mathcal{T}$. The objects of $\mathrm{Cl}(\mathcal{T})$ 
are pairs $(\Gamma . \phi)$ consisting of a finite context $\Gamma=\left(x_{1}: s_{1} ; \ldots ; x_{n}: s_{n}\right)$ of variables $x_{i}$ with assigned types $s_{i}$ and a formula (i.e. by the correspondence between predicates and partial functions discussed above just a definedness assertion) $\phi$ in context $\Gamma$. As (unlike in [26]) we include explicit product types in the language, we often assume that objects are just of the form $(x: s . \phi)$, in order to avoid cluttering the notation. Such an object is thought of as the subtype of $s$ determined by the property $\phi$. Morphisms $\sigma:(\Gamma . \phi) \rightarrow(\Delta . \psi)$ are (type-correct) substitutions of the variables in $\Delta$ by terms in context $\Gamma$ such that $\phi$ entails $\psi \sigma$ as well as definedness of $\sigma(x)$ for every variable $x$ in $\Delta$; morphisms are taken modulo provable equality of terms under $\phi$. In the simplified case where $\Delta$ is of the form $(y: t)$, morphisms can just be regarded as being represented by single terms.

The central facts establishing that the above correspondence is actually an equivalence are that

- the pccc $\mathbf{C}$ is equivalent to $\mathrm{Cl}(\mathrm{Th}(\mathbf{C}))$, and

- the theory $\operatorname{Th}(\mathrm{Cl}(\mathcal{T}))$ is a conservative extension of $\mathcal{T}$.

When reasoning about a pccc with equality, $\mathbf{C}$, one may thus assume that $\mathbf{C}$ is actually of the form $\mathrm{Cl}(\mathrm{Th}(\mathbf{C}))$, i.e. freely move back and forth between logical and categorical arguments, and in particular construct objects in $\mathbf{C}$ as subtypes of types formed from $\mathbf{C}$-objects. It is therefore helpful to recall how some important categorical concepts are reflected in the internal logic:

- Composition is chaining of substitutions.

- The product of types $(\Gamma . \phi)$ and $(\Delta . \psi)$, where the contexts $\Gamma$ and $\Delta$ are w.l.o.g. disjoint, is $(\Gamma ; \Delta . \phi \wedge \psi)$.

- Identities and product projections are just variables.

- The equaliser of two morphisms $(x: s . \phi) \rightarrow(y: t . \psi)$ given by terms $\alpha, \beta$ in context $x: s$ is the type $(x: s . \phi \wedge \alpha \stackrel{e}{=} \beta)$.

The constructions of initial datatypes and final process types in Sect. 5 will be based on this principle. They will, by the above equivalence, amount simultaneously to conservativity results in HASCASL and to existence theorems for datatypes and process types in the categorical semantics. In the latter incarnation, they apply in particular to quasitoposes [36], which may be defined as finitely cocomplete pccc's with equality. This class of categories is technically related to toposes, the essential difference being that the internal logic of a topos speaks about all subobjects, while the internal logic of a quasitopos speaks only about the regular subobjects (or more formally that the classifier of a topos classifies all subobjects, and that of a quasitopos only the regular subobjects).

Remark 1.2. Quasitoposes have a further, first order internal logic which is based on the full subobject fibration. Throughout this work, we use the term 'internal logic of a quasitopos', or more generally of a pccc with equality, to refer to the higher order internal logic based on the regular subobject fibration.

As mentioned above, the range of examples is much broader in the case of quasitoposes; e.g. there are many interesting non-trivial concrete quasitoposes over Set, while concrete toposes over Set are always full subcategories of Set. Intuitively, quasitoposes support a distinction between 'maps', i.e. functional relations, and 'morphisms', i.e. functions, while the two concepts coincide in toposes. Similarly, quasitoposes distinguish between partial maps, designated below by the symbol $\rightarrow$ ?, and single-valued relations. In the internal logic, the difference is captured precisely by the fact that toposes admit unique choice, 
while quasitoposes do not. Objects $A$ in a quasitopos (or in a pccc with equality) that do admit unique choice in the sense described in Sect. 1.1 are called coarse. Explicitly, $A$ is coarse iff there exists a function $c$ from the type $S g(A)$ of singleton subsets of $A$ to $A$ such that $c(p)$ is in $p$ (and hence $p=\{c(p)\}$ ) for every $p: S g(A)$; in this case, the unique description term $\iota x: A \bullet \phi$ can be defined as $c(\lambda x: A \bullet \phi)$.

To give the reader a basic feeling for the above issues, we recall one of the simplest examples of a non-trivial set-based quasitopos, the category ReRe of reflexive relations [1]. The objects of ReRe are pairs $(X, R)$ with $R$ a reflexive relation on the set $X$, and morphisms $f:(X, R) \rightarrow(Y, S)$ are relation-preserving maps $f: X \rightarrow Y$, i.e. $f(x) S f(y)$ whenever $x R y$. We say that $(X, R)$ is discrete if $R$ is equality, and indiscrete if $R=X \times X$. The coarse objects of $\mathbf{R e} \mathbf{R e}$ are precisely the indiscrete objects. The category $\mathbf{R e} \boldsymbol{R e}$ has a natural numbers object, i.e. an initial algebra for the functor $\_+1$, namely the discrete structure on the set of natural numbers. In particular, the natural numbers object fails to be coarse, i.e. does not support unique choice.

\section{Datatypes in HASCASL}

HASCASL supports recursive datatypes in the same style as in CASL [3, 14]. To begin, an unconstrained datatype $t$ is declared along with its constructors $c_{i}: t_{i 1} \rightarrow \ldots \rightarrow t_{i k_{i}} \rightarrow t$ (where the function arrows $\rightarrow$ and $\rightarrow$ ? are right associative) by means of the keyword type in the form

$$
\text { type }::=c_{1} t_{11} \ldots t_{1 k_{1}}|\ldots| c_{n} t_{n 1} \ldots t_{n k_{n}}
$$

(mutually recursive types are admitted as well, but omitted from the presentation for the sake of readability; their handling requires essentially no more than adding more indices). Here, $t$ is a pattern of the form $C a_{1} \ldots a_{r}, r \geq 0$, where $C$ is the type constructor (or type if $r=0$ ) being declared and the $a_{i}$ are type variables. The $t_{i j}$ are types whose formation may involve $C$, the type variables $a_{i}$, and any types declared in the local environment, i.e. the context of preceding declarations. Optionally, selectors $s e l_{i j}: t \rightarrow ? t_{i j}$ may be declared by writing $\left(s_{i j}: ? t_{i j}\right)$ in place of $t_{i j}$. All this is syntactic sugar for the corresponding declarations of types and operations, and equations stating that selectors are left inverse to constructors.

Datatypes may be qualified by a preceding free or generated. The generated constraint introduces an induction axiom; this corresponds roughly to term generatedness ('no junk'). The free constraint ('no junk, no confusion') instead introduces an implicit fold operator, which implies both induction and a primitive recursion principle. If one of these constraints is used, then recursive occurrences (in the $t_{i j}$ ) of $C$ are restricted to the pattern $t=C a_{1} \ldots a_{r}$ appearing on the left hand side; i.e. HASCASL does not support polymorphic recursion. If a free constraint is used, then additionally recursive occurrences of $t$ are required to be strictly positive w.r.t. function arrows, i.e. occurrences in the argument type of a function type are forbidden. We omit a detailed discussion of generatedness constraints [28]. The semantics of freeness constraints is defined in more detail as follows.

Standardly, initial datatypes are characterised by the abovementioned induction axioms (no junk) and additionally by the no confusion condition, stating essentially that all terms formed from the constructors and given elements of the types in the local environment denote distinct values. By the discussion in Sect. 1.3, it is clear that these conditions are insufficient in the setting of HASCASL's internal logic: in the maps vs. morphisms metaphor, they constrain only the underlying set of a datatype, not its structure. E.g. in the quasitopos 
ReRe of reflexive relations, the no-junk-no-confusion axioms for the datatype of natural numbers, i.e. the Peano axioms, will be satisfied by any object whose underlying set is the set of natural numbers. In particular, one will not be able to prove a recursion principle from the Peano axioms (which is possible under unique choice [17]), as models of the Peano axioms in general fail to be initial algebras.

As mentioned above, the semantics of free datatypes in HASCASL is therefore determined by a fold operator, i.e. free datatypes are explicitly axiomatised as initial algebras. As indicated above, recursive occurrences of free types must be strictly positive, i.e. types like $L::=a b s(L \rightarrow L)$ and $L a::=a b s((L \rightarrow a) \rightarrow a)$ are illegal, while

free type Tree a $b::=$ leaf $b \mid$ branch $(a \rightarrow$ Tree $a b)$

is allowed. Free datatypes may thus be seen as initial algebras for functors. In the standard case, the functors in question are polynomial functors, with multiple arguments of constructors represented as products and alternatives represented as sums. E.g. the signature of the tree type above induces the functor $F_{a b}$ given by

$$
F_{a b} c=b+(a \rightarrow c) .
$$

The general mechanism for extracting functors from datatype declaration is explained in more detail in Sect. 3. This mechanism relies on type classes to ensure that user-defined type constructors appearing in constructor arguments are actually functors. The latter will in particular be the case if type constructors are defined as free datatypes with functorial parameters; e.g. the above declaration induces a functor taking $b$ to Tree $a b$.

For now, we take for granted that a free datatype $t$ as in the beginning of this section can be regarded as an initial algebra $\alpha: F t \rightarrow t$ for a functor $F$. Initiality is expressed by means of a polymorphic fold operation

$$
\text { fold }:(F b \rightarrow b) \rightarrow t \rightarrow b
$$

for $b$ : Type, and an axiom stating that, for $d: F b \rightarrow b$, fold $d$ is the unique $F$-algebra morphism from $\alpha$ to $d$, i.e. the unique map $f: t \rightarrow b$ satisfying

$$
d \circ(F f)=f \circ \alpha .
$$

Initiality implies induction and term distinctness, i.e. the usual no-junk/no-confusion conditions: term distinctness follows from the fact that structure maps of initial algebras are isomorphisms (Lambek's lemma); induction for a predicate $P$ on $t$ is proved by applying fold at the type $b=(x: t . P x)$. (The semantics of polymorphism in HAsCAsL prescribes that polymorphic operators such as fold do have instances at subtypes [28]. For polynomial functors, the use of such instances can be circumvented; see Remark 5.7 for more comments on this point.) Moreover, one obtains a primitive recursion principle by means of a simultaneous recursive definition of the identity (as suggested in [6]): The fold operation allows defining recursive functions $f: t \rightarrow b$, where $\alpha: F t \rightarrow t$ is the initial datatype for the functor $F$, using the iteration scheme, i.e.

$$
f(\alpha x)=d(F f x) \text { for } x: F t
$$


(which is just a restatement of the previous equation). One may thus in particular define a function $g: t \rightarrow t \times b$ by

$$
\begin{aligned}
g(\alpha x) & =\left(\lambda y: F(t \times b) \bullet\left(\alpha\left(F \pi_{1} y\right), d y\right)\right)(F g x) \\
& =\left(\alpha\left(F \pi_{1}(F g x)\right), d(F g x)\right) \\
& \left.=\left(\alpha\left(F\left(\pi_{1} \circ g\right) x\right)\right), d(F g x)\right)
\end{aligned}
$$

where $\pi_{1}$ denotes the first projection $\lambda(x, z): t \times b \bullet x$. Here, the actual body of the recursive definition is the map $d: F(t \times b) \rightarrow b$. The defining equation of $g$ implies that $\left.\left(\pi_{1} \circ g\right)(\alpha x)=\alpha\left(F\left(\pi_{1} \circ g\right) x\right)\right)$ and hence, by uniqueness of folds, that the first component $\pi_{1} \circ g$ of $g$ is the identity on $t$. Therefore, $g=\lambda y: t \bullet\left(y,\left(\pi_{2} \circ g\right) y\right)$, so that the second component $f=\pi_{2} \circ g$ of $g$, where $\pi_{2}: t \times b \rightarrow b$ denotes the second projection, satisfies

$$
f(\alpha x)=d(F(\lambda y: t \bullet(y, f y)) x) .
$$

Conversely, every solution $f$ of this equation yields a solution $g=\lambda y: t \bullet(y, f y)$ of the iteration equation for $g$. Thus we may define $f: t \rightarrow b$ by primitive recursive equations, whose right hand side may depend on applications $f x$ of $f$ to constructor arguments $x$ appearing in the pattern $\alpha x$ on the left hand side, as in the case of iteration, and additionally on the constructor arguments $x$ themselves.

Since by Lambek's lemma, the structure map of an initial algebra is an isomorphism, free datatypes $\alpha: F t \rightarrow t$ inherit a case operator from the decomposition of $F t$ as a sum; such an operator

$$
\text { case } x \text { of } c_{1} y_{11} \ldots y_{1 k_{1}} \rightarrow f_{1} y_{11} \ldots y_{1 k_{1}}|\ldots| c_{1} y_{n 1} \ldots y_{n k_{n}} \rightarrow f_{1} y_{n 1} \ldots y_{n k_{n}}
$$

is provided explicitly in HASCASL.

Remark 2.1. Unlike in CASL, the meaning of free type does not coincide with that of the corresponding structured free extension free $\{$ type ... $\}$, which would require all newly arising function types to be also freely term generated.

Example 2.2. Consider the following free datatype definitions.

free type List $a::=$ nil $\mid$ cons $(a$; List $a)$

free type Tree a $b::=$ leaf $a \mid$ branch $(b \rightarrow$ List $($ Tree $a b))$

The declaration of List $a$ induces the standard fold operation for lists. Moreover, the type class mechanism (see Section 3) recognises automatically that the type constructor List is a functor, and in particular generates the standard map operation. For Tree, we obtain a polymorphic fold operation

$$
\text { fold }:(a \rightarrow c) \rightarrow((b \rightarrow \text { List } c) \rightarrow c) \rightarrow \text { Tree } a b \rightarrow c .
$$

This operation is axiomatised as being uniquely determined by the equations

$$
\text { fold } f g(\text { leaf } x)=f x \text { and fold } f g(\text { branch } s)=g(\text { map }(\text { fold } f g) \circ s) \text {. }
$$




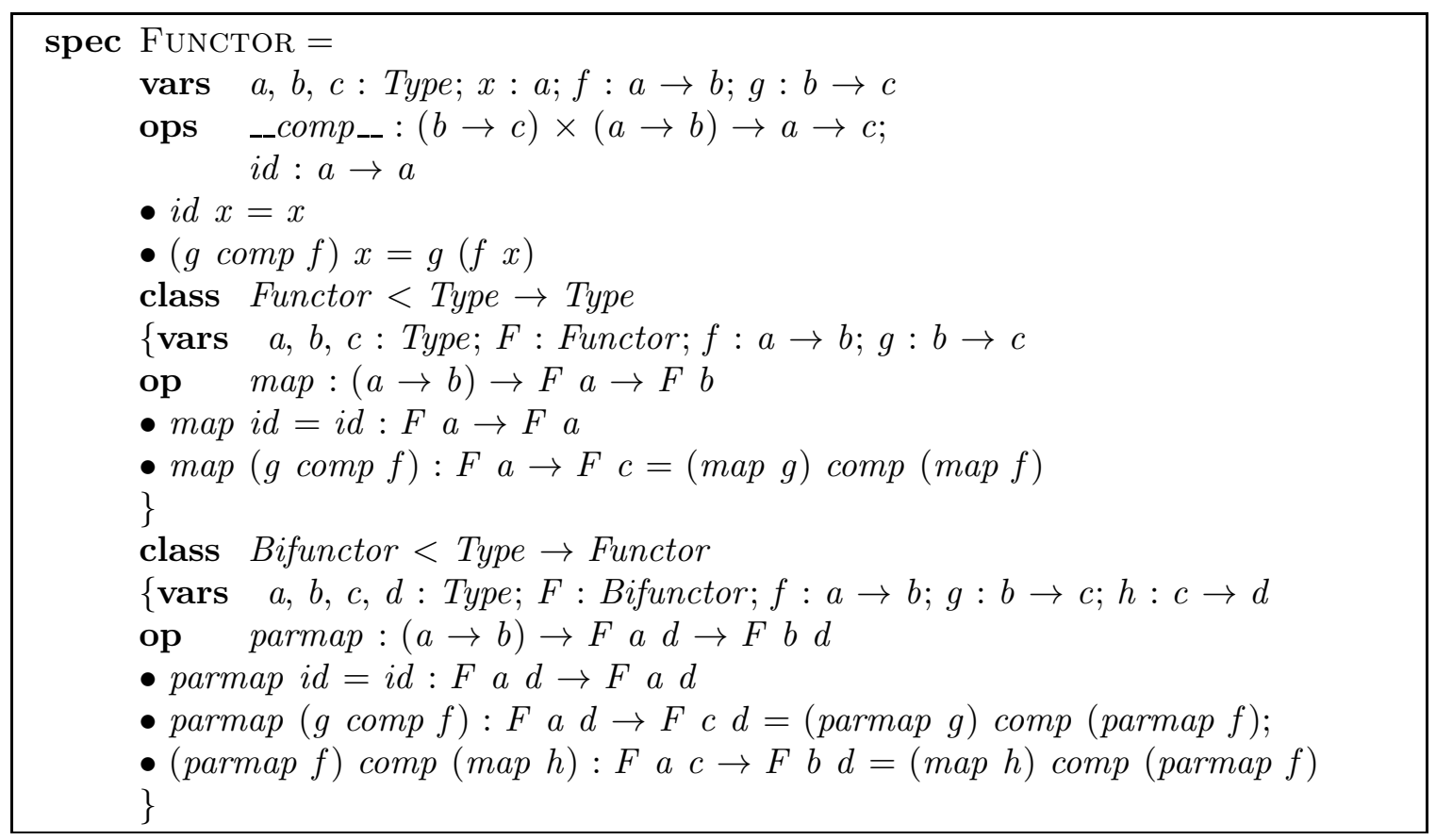

Figure 1: HASCASL specification of functors

\section{Initiality via the Type Class Mechanism}

The concept of free datatype described in the previous section may be regarded as bootstrapped, i.e. as being a HASCASL library equipped with built-in syntactic sugar rather than a basic language feature. The crucial point here is that HASCASL's type class mechanism allows talking about functorial signatures, algebras for a functor, and algebra homomorphisms.

Figure 1 shows the constructor class of functors. Mutually recursive or parametrised datatypes require $n$-ary functors for $n \in \mathbb{N}$, and in fact occasionally type constructors which are functorial only in some of their arguments; since HASCASL does not feature dependent classes, the corresponding classes need to be specified one by one, as exemplified in Fig. 1 by a specification of bifunctors. This is not a problem in practice, as typically only small values of $n$ are needed; the specification of bifunctors illustrates how $n+1$-ary functors can be specified recursively in terms of $n$-ary functors.

Remark 3.1. One might envision a single specification of functors of arbitrary finite arity by abuse of syntax, as follows: declare a class Typelist and type constructors Nil : Typelist, Cons : Type $\rightarrow$ Typelist $\rightarrow$ Typelist, and define Functor as a subclass of Typelist $\rightarrow$ Type. (Undesired semantic side effects may be eliminated by specifying the types Nil, Cons a Nil etc. to be singletons.) Similar tricks work in Haskell [8] but rely on multi-parameter type classes, which are currently excluded from the HASCASL design.

For purposes of conservativity of datatype declarations, the class of polynomial functors (bifunctors etc.), shown in Fig. 2, plays an important role. An $n$-ary functor is polynomial if it can be generated from projection functors (the identity functor if $n=1$ ) and constant functors by taking finite sums and products. These operations, and similar constructions 


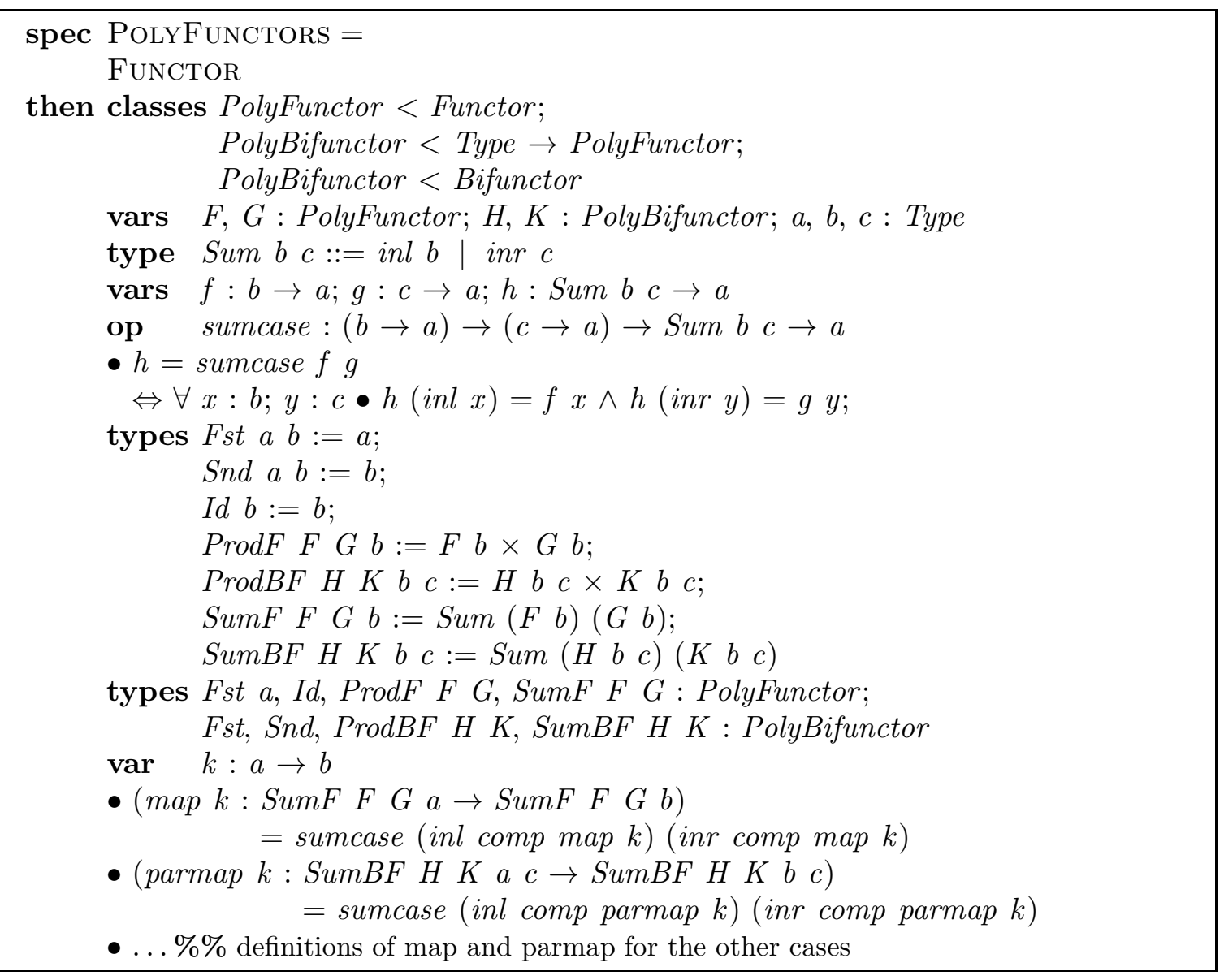

Figure 2: HASCASL specification of polynomial functors

in Fig. 3, are defined as type synonyms, i.e. as mere abbreviations of existing types. ${ }^{1}$ The obvious definitions of the map and parmap operations are omitted for most of the functors introduced in Fig. 2, except in the case of sums. Note that HASCASL does not provide a way to exclude unwanted ('junk') further instance declarations for the class PolyFunctor, i.e. to say that the class is generated by the given generic instances. As in Fig. 1, we show only the specification for functors of arity at most 2; the extension to higher arities is obvious.

In Fig. 3, we present a specification of algebras for a functor. The set of algebra structures for a functor $F$ on a type $a$ is given by the type constructor $A l g$, which depends on both $F$ and $a$ and thus has the profile Functor $\rightarrow$ Type $\rightarrow$ Type; it is given as a type synonym for the type $F a \rightarrow a$. Similarly, the type constructor AlgMor for algebra morphisms depends on $F$ and types $a, b$ forming the carriers of the domain and the codomain, respectively. Algebra morphisms are treated as triples consisting of two algebra structures and a

\footnotetext{
${ }^{1}$ Consequently, the specifications, while correct according to the HASCASL language definition, fail to pass the static analysis in the present version of the heterogeneous tool set Hets 12, as type synonyms are currently immediately expanded and $\beta$-reduced; this will be remedied in future versions of the tool. In [27, we have used type declarations with explicit constructors as a workaround in place of type synonyms; for purposes of the present work, we have given preference to readability of specifications.
} 


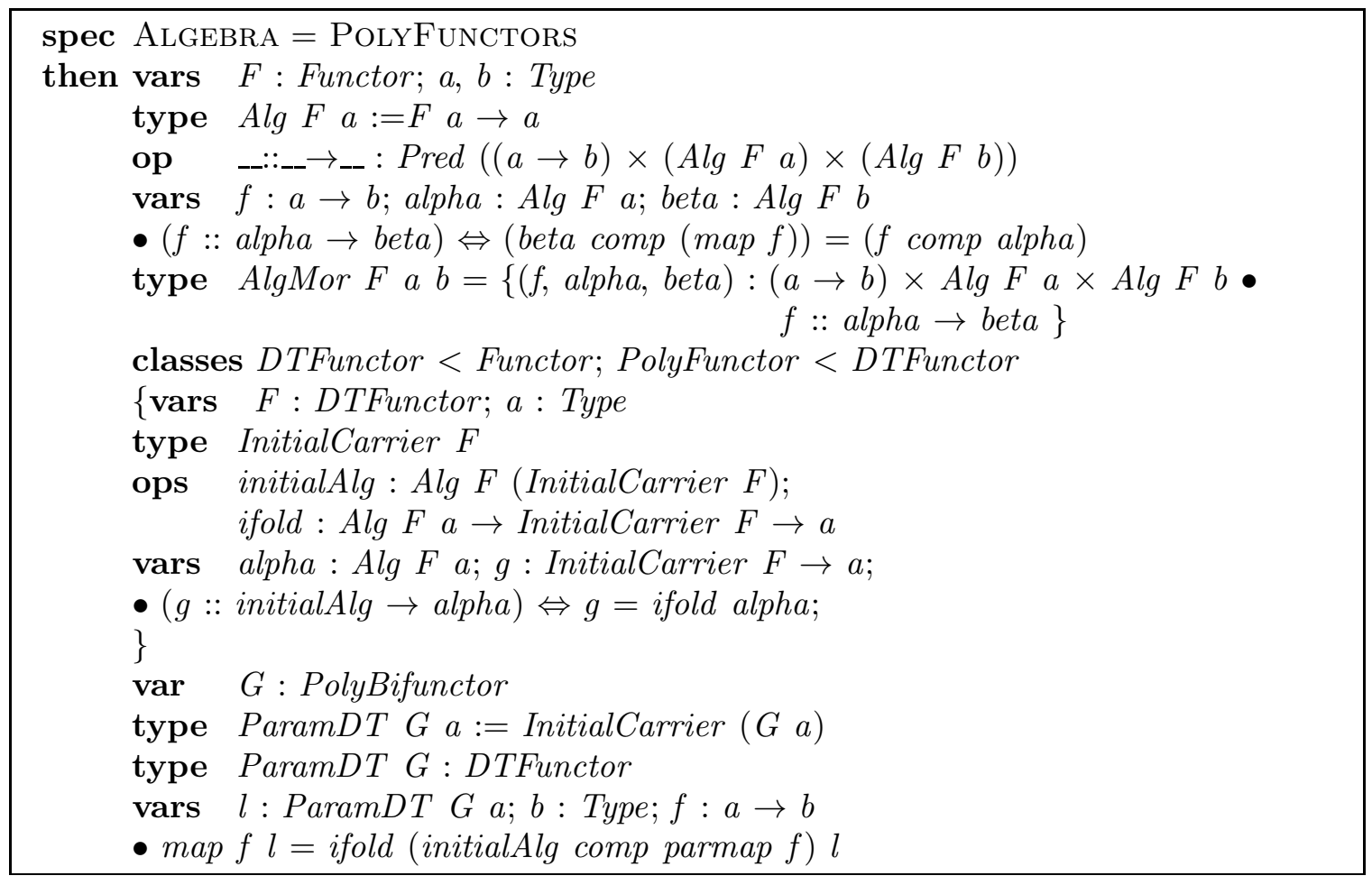

Figure 3: HASCASL specification of initial algebras

map between the carriers, thus circumventing the absence of dependent types - such as the 'type' of algebra morphisms between algebras alpha and beta - in HASCASL (these can be conservatively added to the language [25], however at the price of making type checking undecidable).

Initial algebras are then specified by means of two operations: a type constructor InitialCarrier that assigns to a functor the carrier set of its initial algebra, and a polymorphic constant initialAlg which represents the structure map of an initial algebra for $F$ on this carrier. Initiality of this algebra is specified by means of an explicit fold operation, called ifold in the specification. As initial algebras will exist only for some functors, the abovementioned operations are defined only on a subclass DTFunctor ('datatype functor') of Functor. We declare the class PolyFunctor (Fig. 2) to be a subclass of DTFunctor, thus stating that all polynomial functors have initial algebras as proved in Sect. 5 , due to possible junk in the class PolyFunctor (see above), this is consistent but non-conservative. Moreover, we state that initial algebras depend functorially on parameters in the case of polynomial bifunctors and that the arising functor again has an initial algebra (as nested recursion may be coded by mutual recursion in the standard way [7]) by defining a type constructor ParamDT which maps a polynomial bifunctor $G$ to the functor that takes a type $a$ to the initial algebra of the polynomial functor $G$ a, and by declaring ParamDT $G$ to be an instance of DTFunctor.

Remark 3.2. Note that functors induced by parametrised initial datatype declarations are declared as instances of DTFunctor in Fig. 3 only if the signature functor is polynomial. It is not possible to extend this mechanism to arbitrary parametrised datatypes, as the 
corresponding functors need not themselves have initial algebras. As a simple example, consider the declaration

$$
\begin{aligned}
& \text { var } a: \text { Type } \\
& \text { free type } C a::=a b s(\text { Pred } a)
\end{aligned}
$$

which defines $C a$ as the initial algebra of the functor $F$ given by $F a b=$ Pred $a$, i.e. $F$ takes the powerset of its first argument and ignores the second. Thus, $C a$ is isomorphic to Pred $a$ and hence, by Russell's paradox, the functor $C$ does not have an initial algebra.

We conclude with a brief description of how the data above are generated by the static analysis of actual HASCASL specifications. The functor $F$ associated to the declaration of a datatype $t$ as in the beginning of Sect. 2 is a sum of $n$ functors $F_{i}$, one for each constructor $c_{i}$; the functor $F_{i}$, in turn, is a product of $k_{i}$ functors $F_{i j}$, corresponding to the $t_{i j}$. The $t_{i j}$ are, by the restrictions laid out in Sect. 2, inductively generated from types in the local environment, $t=C a_{1} \ldots a_{n}$, and the type variables $a_{i}$ by taking products, exponentials $s \rightarrow t$ or $s \rightarrow$ ? $t$, where $s$ is a type formed from the $a_{i}$ and the local environment, and applications $D s_{1} \ldots s_{l}$ of type constructors from the local environment, the latter subject to the restriction that if $s_{i}$ contains a recursive occurrence of $t$, then the dependence of $D$ of its $i$-th argument must be functorial. The latter property is tracked by means of the type class mechanism; in particular, instances of Functor are generated automatically for parametrised datatypes such as the type List a of Example 2.2. Given this format of the $t_{i j}$, it is straightforward to associate a functor to each $t_{i j}$ (using further generic instances of Functor, in particular exponentials and closure under functor composition). Finally, an instance $F$ : DTFunctor is generated. If this instance is already induced by the generic instances shown in Fig. 3, then the datatype declaration is guaranteed to be conservative (see Section 51); otherwise, conservativity and in fact consistency of the datatype declaration becomes the responsibility of the user. This happens in particular when constructor arguments involve either type constructors from the local environment which are not themselves declared as initial datatypes or exponentiation with types from the local environment. Whether or not datatype declarations are conservative in the latter case, which in particular includes the case of infinite branching, remains an open problem; under unique choice, declarations of infinitely branching datatypes are conservative [10, 18, 2]. If $F$ is moreover of the class PolyBifunctor (or a corresponding class of functors of higher arity), then an instance $C:$ DTFunctor is generated.

Using the sumcase operation of Fig. 2, one can gather the constructors of $t$ into a structure map $c: F t \rightarrow t$; the freeness constraint then translates into the declaration of a two-sided inverse $g$ of ifold $c$. The fold operation on $t$ is obtained as fold $\alpha=($ ifold $\alpha) \circ g$.

\section{Process Types in HasCasl}

Although process types in the style of CoCASL, so-called cotypes [13, are not presently included in the HASCASL design, the results of the previous section indicate that cotypes could be integrated seamlessly into HASCASL. A cotype is a syntactic representation of a coalgebra for a signature functor. Cotypes are declared in a similar style as types; the crucial difference is that, while selectors are optional in a datatype, they are mandatory in a cotype, as they constitute the actual structure map of the coalgebra, and constructors are optional. Thus, the core of a cotype declaration has the form 
cotype $t::=\left(\operatorname{sel}_{11}: t_{11} ; \ldots ; \operatorname{sel}_{1 k_{1}}: t_{1 k_{1}}\right)|\ldots|\left(\operatorname{sel}_{n 1}: t_{n 1} ; \ldots ; \operatorname{sel}_{n k_{n}}: t_{n k_{n}}\right)$ where $t$ is a pattern $C a_{1} \ldots a_{n}$ consisting of a newly declared type constructor $C$ and type variables $a_{1}, \ldots, a_{n}$. A cotype induces axioms guaranteeing that the domains of selectors in the same alternative agree, and that the domains of all alternatives form a coproduct decomposition of the cotype. Thus, e.g. the models of the cotype

cotype Proc $::=($ out :? a; next :? Proc) | (spawnl, spawnr : Proc)

are coalgebras for the functor $F$ given by $F X=a \times X+X \times X$. The semantics of cotypes, in particular cofree (i.e. final) cotypes, builds on a dual of the specification of algebras (Fig. (3)), where the type of algebras is replaced by a type Coalg $F a:=a \rightarrow F a$, the definition of homomorphisms is correspondingly modified, and initiality is replaced by finality, i.e. unique existence of morphisms into the final coalgebra given by an unfold operation. For cofree cotypes, the codomains of the selectors are, as in the case of initial datatype, required to depend functorially on $t$; of course, this will not in general guarantee existence of final coalgebras. The extraction of functors from cotype signatures is analogous to the case of types as explained in Sect. 3, with two differences:

- the class of functors that admit final coalgebras contains a generalised class of polynomial functors that allows replacing identity functors by exponentiation with constant exponents (see Section (5);

- unlike for free types, functors arising from cofree cotype declarations, even for polynomial functors, typically do not have final coalgebras.

We omit the discussion of cogeneratedness of cotypes.

The only subtle point in the matching between cotype declarations in HASCASL and coalgebras is that the conditions imposed in CoCASL to ensure that a cotype $t$ with associated functor $F$ decomposes as a disjoint union of the domains of the selectors would in HASCASL be insufficient to guarantee existence of a single structure map $t \rightarrow F t$, the point being, again, the absence of unique choice. As indicated above, we thus impose, instead of just disjointness and joint exhaustiveness of the domains, that the cotype is the coproduct of the domains, by introducing a polymorphic partial case operation similar to the sumcase operation of Fig. 2, E.g. for the cotype Proc above and $f, g:$ Proc $\rightarrow$ ? $a$, case $f g=h:$ Proc $\rightarrow a$ is defined whenever the domains of $f$ and $g$ equal the domains of out and spawnl, respectively, and in this case $h$ extends $f$ and $g$. (Under unique choice, case $f g$ is definable as $\lambda p: \operatorname{Proc} \bullet \iota x: a \bullet x=f(p) \vee x=g(p)$.)

\section{Conservativity of Datatypes and Process Types}

Free datatypes in HASCASL are not necessarily conservative extensions of the local environment. Already the naturals may be non-conservative: as discussed in Sect. 1, conservative extensions can only introduce names for entities already in the present signature, and a given model might interpret all types as finite sets. This problem arises already in standard HOL, where the construction of initial datatypes [18, 2] is based on the naturals. The constructions given in [18, 2] make heavy use of unique choice, so that the question arises whether similar constructions are possible in HASCASL. Below, we answer this question in the affirmative for the case of finitely branching datatypes; it remains open for the infinitely branching case. By the equivalence of HASCASL with the internal logic of partial cartesian closed categories with equality (Sect. 1.3), our results extend to pccc's with equality and finite coproducts, and hence in particular to quasitoposes. 
To begin, we fix the required additional infrastructure. As seen above, already the construction of signature functors for standard datatypes requires finite sums. These are specified (non-conservatively) in HASCASL by declaring a type constructor Sum as in Fig. 2, and moreover an initial type Zero which is specified as having a function zero: Zero $\rightarrow a$ into every type $a$ and satisfying the axiom $\forall x:$ Zero. $\perp$, where we use $\top$ and $\perp$ to denote truth and falsity, respectively. This axiom means that the type Zero is uninhabited, and implies that for every type $a$, zero is the only function Zero $\rightarrow a$. Below, we denote sums by + and the initial type by 0 in the interest of readability, with injection functions inl : $A \rightarrow A+B$ and inr : $B \rightarrow A+B$ as usual; moreover, we use the standard case notation as discussed in the case of initial datatypes in Sec. 2, and denote the unit type Unit by 1 . We write Bool for the sum type $1+1$, denoting the associated case operator as if - then - else and the terms inl () and inr () as true and false, respectively. We refer to the extension of the partial $\lambda$-calculus with equality by,+ 0 , and the associated operators and axioms as the partial $\lambda$-calculus with equality and sums.

Remark 5.1. In a cartesian closed category, finite coproducts are always internal in the sense that copairing is embodied in an operation (sumcase in the above notation) which satisfies the relevant laws (Fig. 2) internally [20]. Thus, the internal language $\operatorname{Th}(\mathbf{C})$ of a pccc $\mathbf{C}$ with equality and finite coproducts has sum types as specified in Fig. 2. It is moreover clear that $\operatorname{Th}(\mathbf{C})$ has a type Zero $=0$, operations $0 \rightarrow a$ for every type $a$, and an axiom $\forall x: Z$ ero. $\perp$, the latter because the unique morphism $0 \rightarrow 1$ in $\mathbf{C}$ equalises the truth values $T, \perp: 1 \rightarrow$ Logical. Conversely, the classifying category of a theory with sums has finite coproducts: one has to check that the binary coproduct of objects $(x: a . \phi),(y: b . \psi)$ exists in the classifying category; but this is just the object

$$
(z: a+b \text {. case } z \text { of inl } x \rightarrow \phi \mid \text { inr } y \rightarrow \psi) \text {. }
$$

Similarly, one easily checks that 0 has a unique morphism into every object $(x: a . \phi)$ of the classifying category. It follows that

the partial $\lambda$-calculus with equality and sums is the internal logic of pccc's with equality and finite coproducts.

The search for the internal logic of quasitoposes, i.e. a logic that would be equivalent to quasitoposes via an internal language/classifying category correspondence, remains open. Recall that a quasitopos is a pccc with equality and finite colimits, i.e. finite coproducts and coequalisers. Hence, the missing ingredient is a suitable logical representation of coequalisers, i.e. quotients. We conjecture that the key to this is a generalisation from subtypes $(x: a . \phi)$ to subtypes with replacement, i.e. types of the form $(f(x) . x: a ; \phi)$, representing the quotient of $(x: a . \phi)$ by the kernel of a function $f: a \rightarrow b$.

Unlike in toposes, coproducts in quasitoposes, and hence in pccc's with equality, need not be disjoint; i.e. the pullback of distinct coproduct injections need not be the initial object. Specifically, one has

Proposition 5.2. Let $\mathbf{C}$ be a pccc with equality. Then $\mathbf{C}$ has disjoint finite coproducts iff $\mathbf{C}$ has a (strict) initial object 0 , the coproduct $1+1$ exists in $\mathbf{C}$, and the monomorphism $0 \rightarrow 1$ is regular.

The proof needs the following observation.

Lemma 5.3. If $0 \rightarrow 1$ is regular in a pccc with equality, then every type a has a partial constant bot $: 1 \rightarrow$ ? a such that $\neg \operatorname{def}($ bot ()$)$. 
Proof. The assumption implies that 0 is isomorphic to the object $(x: 1 . \perp)$. Thus we can put bot $x=z(x\lceil\perp)$, where $z$ is the unique morphism $(x: 1 . \perp) \cong 0 \rightarrow a$.

Proof (Proposition 5.2). 'Only if' holds universally: If coproducts are disjoint, then $0 \rightarrow 1$ is a regular monomorphism, being the pullback e.g. of the left injection $1 \rightarrow 1+1$, which is even a section. Moreover, initial objects in cartesian closed categories are always strict: since the functor $-\times A$ is left adjoint for every $A$, it preserves the initial object 0 , i.e. $A \times 0 \cong 0$; this is easily seen to imply that every object $B$ that has a morphism $B \rightarrow 0$ is initial.

'If': Given Bool $=1+1$ with operations false, true, and if - then - else as above, one can construct the binary sum $a+b$ of objects $a, b$ in $\mathbf{C}$ as

$$
(x: 1 \rightarrow ? a, y: 1 \rightarrow ? b, z: \text { Bool. }(\operatorname{def}(x()) \Leftrightarrow z=\text { true }) \wedge(\operatorname{def}(y()) \Leftrightarrow z=\text { false })) .
$$

The coproduct injections are given by inl $x=((\lambda z: 1 \bullet x),(\lambda z: 1 \bullet$ bot ()$)$, true $)$, and inr $y=((\lambda z: 1 \bullet$ bot ()$),(\lambda z: 1 \bullet y)$, false), with bot according to Lemma 5.3. The copairing $h=$ sumcase $f g$ of functions $f: a \rightarrow c, g: b \rightarrow c$ is then defined as

$$
h(x, y, z)=\text { if } z \text { then } f(x()) \text { else } g(y()) \text {. }
$$

It is easy to see that the copairing is uniquely determined.

This proves existence of finite coproducts; it remains to prove that coproducts are disjoint. One checks by an easy diagram chase that if 0 is strict and $1+1$ is disjoint, then every coproduct is disjoint. It is moreover easy to see that if the monomorphism $0 \rightarrow 1$ is regular, then $1+1$ is disjoint.

Remark 5.4. The previous statement means in particular that coproducts in a pccc with equality and finite coproducts, in particular in a quasitopos, are disjoint iff the monomorphism $0 \rightarrow 1$ is regular. This may but need not be the case. Positive set-based examples include the above-mentioned quasitoposes of pseudotopological spaces and (reflexive) relations, respectively, and more generally any set-based quasitopos whose forgetful functor preserves pullbacks and induces a singleton fibre over the empty set. The simplest example where $0 \rightarrow 1$ is not regular are Heyting algebras, which are quasitoposes when regarded as thin categories: in these, 0 is the bottom element, and 1 is the top element, but the only regular monomorphisms are the isomorphisms, so that $0 \rightarrow 1$ fails to be regular except in the degenerate case. Note that the Heyting algebras are, up to equivalence of categories, precisely the quasitoposes with an inconsistent internal logic, i.e. with $T=\perp$. An example with a consistent internal logic is the following. Let $\operatorname{Spa}(\mathcal{P})$ be the category whose objects are pairs $(X, \mathcal{A})$ with $X$ a set and $\mathcal{A} \subseteq \mathcal{P}(X)$, and whose morphisms $(X, \mathcal{A}) \rightarrow(Y, \mathcal{B})$ are maps $f: X \rightarrow Y$ such that for all $A \in \mathcal{A}, f[A] \in \mathcal{B}$. By results of [1] (or easy direct verification), $\operatorname{Spa}(\mathcal{P})$ is a quasitopos. However, $0 \rightarrow 1$ is not regular: 0 is the object $(\emptyset, \emptyset)$, while 1 is the object $(\{*\}, \mathcal{P}(\{*\}))$ (and hence the regular subobject $(z: 1 . \perp)$ is $(\emptyset,\{\emptyset\})$, not $(\emptyset, \emptyset))$.

Remark 5.5. The undefined constant bot of Lemma 5.3 can be used e.g. to define partial extraction functions outl $: a+b \rightarrow$ ? a, outr : $a+b \rightarrow$ ? $b$ by outl $z=$ case $z$ of inl $x \rightarrow$ $x \mid$ inr $y \rightarrow$ bot (), analogously for outr. (This implies moreover that $a$ and $b$ are regular subobjects of $a+b$.) Conversely, it should be noted that unless coproducts are disjoint, types $1 \rightarrow$ ? a may fail to have closed inhabitant terms, and extraction functions need not exist. Some constructions in the preliminary version of this work [27] erroneously made use of bot without identifying disjointness of coproducts as an additional assumption. The 
main results, however, remain correct also without this assumption, as we show below; occasionally, this requires slightly unexpected workarounds.

Remark 5.6. Under unique choice, $B o o l=1+1$ coincides with the type

$$
(p: \text { Logical. } p \vee \neg p),
$$

with injections inl ()$=\top$ and $i n r()=\perp$. The copairing $h=$ sumcase $f g$ of two functions $f, g: 1 \rightarrow a$ is then defined as $h p=\iota x: a \bullet(p \Rightarrow f()=x) \wedge(\neg p \Rightarrow g()=x)$. Moreover, it is easy to see that, under unique choice, the object $(z: 1 . \perp)$ is initial. By Proposition 5.2, this reproves the well-known fact that toposes have disjoint finite coproducts. In a quasitopos, one cannot in general construct Bool as a subtype of Logical - the latter is typically an indiscrete space, while Bool is typically discrete. E.g. in the quasitopos of reflexive relations, Logical carries the universal relation, while Bool carries the equality relation.

As indicated above, we shall also need the standard notion of natural numbers object (nno). Categorically, an nno is an initial algebra for the functor _+ + ; in HASCASL, a corresponding type of natural numbers is specified as

free type $N a t::=0$ | suc $N a t$

Remark 5.7. In a cartesian closed category, every nno is internal in the sense that the unique existence of an algebra morphism from the nno into a given -+1 -algebra holds as a formula of the internal logic and is embodied by an operation, fold in the above notation [20]; the same holds for initial algebras, and dually for final coalgebras, of arbitrary strong functors, in particular polynomial functors. The explicit distinction of internal nno's used in [27] is thus superfluous. It follows that the internal language of a pccc with equality, sums, and nno always has a type Nat as specified above. Conversely, the classifying category of a theory with sums and the type $N a t$ will have $(n: N a t . \top)$ as an nno. To see this, one has to show that the fold operation applies also to $\_+1$-algebras on subtypes $(x: a . \phi)$. This can be proved without resorting to instances of fold at such subtypes, as indicated in Sect. 2, as announced there, the argument presented in the following is general enough to apply to arbitrary polynomial functors. The relevance of this point is that assuming instances of fold at subtypes essentially amounts to postulating induction as a separate axiom, rather than deriving it from recursion.

To begin, note that the induction principle on Nat may be proved using fold only at the type Logical: given a predicate $P$ on $N a t$ such that $P 0$ and $\forall n: N a t \bullet P n \Rightarrow P($ suc $n)$, define a predicate $Q$ on $N a t$ recursively by

$$
\begin{aligned}
Q 0 & =P 0 \\
Q(\text { suc } n) & =Q n \wedge P(\text { suc } n) .
\end{aligned}
$$

Then $Q$ has the defining property of fold $g$, where $g:$ Logical $+1 \rightarrow$ Logical is the copairing of the identity and $T$. As the constantly true predicate on Nat also has this property, it follows that $Q$ and hence $P$ hold universally.

Then, a morphism $d:(x: a . \phi)+1 \rightarrow(x: a . \phi)$ induces in the obvious way a morphism $d^{?}:((1 \rightarrow ? a)+1) \rightarrow(1 \rightarrow ? a)$. One thus obtains $f=$ fold $d^{?}: N a t \rightarrow(1 \rightarrow ? a)$. It remains to show that $f$ factors through $(x: a . \phi)$, i.e. that $f n$ is defined and satisfies $\phi[f n / x]$ for all $n$; this is proved by induction.

We have thus established that

the partial $\lambda$-calculus with equality, sums, and Nat is the internal language of pccc's with equality, finite coproducts, and nno. 
We shall now prove the existence of initial algebras and final coalgebras for certain classes of functors the categorical semantics, thus partially generalising known results for $W$-types in toposes (e.g. [10]).

Definition 5.8. The class of polynomial functors is inductively generated from the identity functor and constant functors by taking finite sums and products. The class of extended polynomial functors is inductively generated from the exponential functors with constant exponent (including the identity functor by taking exponent 1) and constant functors by taking finite sums and products.

Of course, the intended interpretation of the constructor class PolyFunctor from Sect. 3 is the class of polynomial functors, and correspondingly for the more general constructor class appearing in the semantics of cotypes (Sect. 4) and the class of extended polynomial functors.

Theorem 5.9. Let $\mathbf{C}$ be a pccc with equality, finite coproducts, and nno (e.g. a quasitopos with nno). Then

(a) C has initial algebras for polynomial functors;

(b) $\mathbf{C}$ has final coalgebras for extended polynomial functors.

The constructions employed in the proof are essentially subtype definitions in the internal logic. By the discussion in Sect. 1.2 and 1.3, it follows that, as an extension of the specification of sums and the natural numbers, the declaration of a datatype $t=C a_{1} \ldots a_{n}$ with constructor arguments $t_{i j}$ as in the beginning of Sect. 2 is conservative, provided that the $t_{i j}$ are built from $t$, the $a_{i}$, and types from the local environment using only product and sum type formation. Moreover, the declaration of a final process type $t=C a_{1} \ldots a_{n}$ as in Sect. 4 is conservative as an extension of the specification of sums and the natural numbers, provided that the codomains $t_{i j}$ of the selectors are built from $t$, the $a_{i}$, and types from the local environment using only product and sum type formation and exponentiation with exponents not depending on $t$. Since the class of pccc's with equality, finite coproducts, and nno is easily seen to be stable under taking products of categories, Theorem 5.9 implies moreover that analogous results hold for declarations of several mutually recursive types or cotypes, respectively.

We begin by proving the existence of a particular datatype, the type of lists:

Lemma and Definition 5.10. Let $\mathbf{C}$ be a pccc with equality, finite coproducts, and nno. Then $\mathbf{C}$ has list objects, i.e. for every object $A$, the functor $1+A \times_{-}$has an initial algebra, the type of lists over $A$.

Proof. We construct the type List of lists over $a$ as

$$
\text { List }=1+(l: N a t \rightarrow ? \text { A, } n: N a t . \forall m: N a t \bullet \operatorname{def}(l m) \Leftrightarrow m \leq n)
$$

(where $\leq$ is defined recursively). We define the list constructors nil : List and cons : $A \rightarrow$ List $\rightarrow$ List by nil $=$ inl () and

$$
\begin{array}{r}
\text { cons } x l=\text { case } l \text { of } \operatorname{inl}() \rightarrow \operatorname{inr}((\lambda k: \text { Nat } \bullet \text { case } k \text { of } 0 \rightarrow x \mid \text { suc } m \rightarrow x \uparrow \perp), 0) \\
\qquad \operatorname{inr~}(l, n) \rightarrow \operatorname{inr}((\lambda k \bullet \text { case } k \text { of } 0 \rightarrow x \mid \text { suc } m \rightarrow l m) \text {, suc } n) .
\end{array}
$$

Given a further $1+A \times$-algebra $B$ with operations $c: B$ and $f: A \times B \rightarrow B$, the folded function $g=$ fold $c f:$ List $\rightarrow B$ is defined by $g z=$ case $z$ of inl ()$\rightarrow c \mid$ inr $(l, n) \rightarrow h l n$, 
where $h$ is defined by recursion over $N a t$ :

$$
\begin{aligned}
h l 0 & =f(l 0) c \\
h l(\text { suc } n) & =f(l \quad 0)(h(\lambda k: N a t \bullet l(\text { suc } k)) n) .
\end{aligned}
$$

It is easy to check that $g$ satisfies, and is uniquely determined by, the defining equation for fold $c f$. Moreover, as recursion on natural numbers is embodied as an operation, so is the recursion principle on lists.

Note how in the construction of the list datatype, an explicit list length component serves to enable inheritance of the recursion operator from the natural numbers. This principle is also at the heart of the general construction of datatypes below, where we employ an explicit depth component on trees. Note moreover that this component is not needed in the construction of final coalgebras.

A maybe slightly unexpected feature of the construction, which illustrates the points made in Remark 5.5, is the fact that we need to treat the empty list as a special case - in general, we cannot code it as the everywhere undefined function $N a t \rightarrow$ ? A, as the latter may fail to exist. Singleton lists, on the other hand, are unproblematic: once we have an element $x: A$ of the list in hand, we obtain an undefined term of type $A$ as $x \uparrow \perp$.

Proof of Theorem 5.9. By Remark 5.7, we can conduct the proof in the classifying category of the internal language of $\mathbf{C}$, the latter being a partial $\lambda$-theory with equality, finite sums, and nno.

(a): We can assume that the given functor $F$ is of the normal form $F X=\sum_{i=1}^{n} A_{i} \times X^{k_{i}}$ with $k_{i} \in \mathbb{N}$ and constant parameter objects $A_{i}$. Moreover, by collecting all $A_{i}$ with $k_{i}=0$ into a single sum type ( 0 in case $k_{i}>0$ for all $i$ ), we may assume that $k_{i}=0$ iff $i=1$, so that $A_{1}$ may be thought of as the type of constants in the signature. Let $A=\sum_{i=1}^{n}\left(A_{i}+1\right)$, with injections into the outer coproduct denoted $i n_{i}$, and injections into the inner coproducts denoted inl, inr as usual. Let Path be the type of lists of natural numbers (which exists in $\mathbf{C}$ according to Lemma 5.10), with constructors nil : Path, cons : Nat $\rightarrow$ Path $\rightarrow$ Path. We now define a universal type of trees, from which the desired initial algebra will be carved out as a subtype, by

$$
\text { DTree }=\left(l: \text { Path } \rightarrow ? \text { A; } d: \text { Path } \rightarrow ? \text { Nat; } x: A_{1}\right),
$$

where for $(l, d, x): D$ Tree and $p: P a t h, l p=i n_{i} z$ indicates that the subtree at $p$ is either a leaf labelled $y$, if $z=i n l y$, or a node labelled by the $i$-th constructor, if $z=i n r()$, and $d p=n$ indicates that the subtree at $p$ has depth $n$. The third component $x: A_{1}$ is a dummy that serves only to enable the construction of undefined terms (see Remark 5.5). We put depth $(l, d, x)=d$ nil for $(l, d, x):$ DTree, and for $j: N a t, j>0$, we define a generic $j$-th selector by $\operatorname{sel}_{j}(l, d, x)=(l \circ($ cons $j), d \circ($ cons $j), x)$. Moreover, we define generic constructors $c_{i}: A_{i} \times D$ Tree $^{k_{i}} \rightarrow D$ Tree, thus making DTree into an F-algebra, by

$c_{i}\left(y,\left(l_{1}, d_{1}, x_{1}\right), \ldots,\left(l_{k_{i}}, d_{k_{i}}, x_{k_{i}}\right)\right)= \begin{cases}\left(l, d, x_{1}\right) & \text { if } k_{i}>0 \\ (l, d, y) & \left.\text { if } k_{i}=0 \text { (and hence } i=1, \text { so that } y: A_{1}\right)\end{cases}$

where $l$ and $d$ are defined by case distinction as

$$
\begin{aligned}
l \text { nil } & =i n_{i}(\operatorname{inr}()) & l(\text { cons } j p) & =\left(\text { if } j=0 \text { then } i n_{i}(\text { inl } y) \text { else } l_{j} p\right) \\
d \text { nil } & =1+\max \left(d_{1} \text { nil }, \ldots, d_{k_{i}} \text { nil }\right) & d(\text { cons } j p) & =\left(\text { if } j=0 \text { then } 0 \text { else } d_{j} p\right) .
\end{aligned}
$$


Here, the maximum is defined by recursion on the naturals, with $\max ()=0$; and the if expressions abbreviate obvious case expressions. The expressions denoted for the sake of readability as $l_{j} p$ and $d_{j} p$ on the right hand side in reality abbreviate long case distinctions over $j=1, \ldots, j=k_{i}, j>k_{i}$, with $l_{j} p$ and $d_{j} p$ undefined for $j>k_{i}$. E.g. for $l_{j}$, we have

$$
l_{j} p= \begin{cases}b_{A} y & \text { if } j=0 \text { or } j>k_{i} \\ l_{1} p & \text { if } j=1 \\ \ldots & \\ l_{k_{i}} p & \text { if } j=k_{i}\end{cases}
$$

where $b_{\text {ot }} y=\left(i n_{i} y\right) \uparrow \perp$, and the case distinction can be emulated by a finite chain of $0 /$ suc case statements.

We then take the carrier $T$ of the desired initial algebra to be the smallest subtype of $D$ Tree closed under the $c_{i}$; thus, $T$ inherits from $D$ Tree the structure of an $F$-algebra. Note that for all $(l, d, x): T$, depth $(l, d, x)>0$ and $l$ nil $=i n_{i}(i n r())$ for some $i$. We have to show that we can construct the function fold $b_{1} \ldots b_{n}: T \rightarrow B$ for functions $b_{i}$ constituting an $F$-algebra on a type $B$. We define a primitive recursive function $f: N a t \rightarrow T \rightarrow$ ? $B$ by

$$
\begin{gathered}
f 0(l, d, x)=\text { bot }_{B} x \\
f(\text { suc } n)(l, d, x)=\text { case } l \text { nil of }_{\left(i n_{i} z \rightarrow \text { case } z \text { of inl } y \rightarrow \text { bot }_{B} x\right.} \\
\mid \operatorname{inr}() \rightarrow \text { case l }[0] \text { of } \\
\text { in } n_{i} y \rightarrow \text { case } y \text { of inr }() \rightarrow \text { bot }_{B} x \\
\text { inl } w \rightarrow b_{i}\left(w, f n\left(\operatorname{sel}_{1}(l, d, x)\right), \ldots, f n\left(\operatorname{sel}_{k_{i}}(l, d, x)\right)\right) \\
\text { otherwise } \left.\rightarrow \operatorname{bot}_{B} x\right)_{i=1, \ldots, n},
\end{gathered}
$$

where otherwise is a placeholder for all remaining cases in a case statement, in this case $i n_{j} y$ for $j \neq i, \operatorname{bot}_{B} x=\left(b_{1} x\right) \uparrow \perp$, and [0] = cons 0 nil. Finally, put

$$
\text { fold } b_{1} \ldots b_{n} z=f(\text { depth } z) z \text {. }
$$

One verifies directly from the defining equations for $f$ that this definition satisfies the fold equation. Moreover, one shows using the definition of $T$ as the least subtype of DTree closed under the constructors that fold $b_{1} \ldots b_{n}$ is total (i.e. one never runs into the exceptional cases $\operatorname{bot}_{B} x$ in the above definition of $f$ ), and uniquely determined by the fold equation.

(b): We can assume that the given functor $F$ has the normal form $F X=\sum_{i=1}^{n} A_{i} \times$ $\left(B_{i} \rightarrow X\right)$, with constant parameter objects $A_{i}, B_{i}$ : it is easy to see that the class of functors isomorphic to such normal forms contains all exponential functors and all constant functors (noting that parameter objects can also be 1 or 0 ) and is closed under sums; to see closure under products, note that a product of two such normal forms is a sum of summands of the form $(A \times(B \rightarrow X)) \times\left(A^{\prime} \times\left(B^{\prime} \rightarrow X\right)\right)$. Such a summand is isomorphic to $\left(A \times A^{\prime}\right) \times\left(\left(B+B^{\prime}\right) \rightarrow X\right)$.

Now put $A=\sum_{i=1}^{n} A_{i}$ and $B=\sum_{i=1}^{n} B_{i}$, with injections $i n_{i}$ in both cases. Define Path as the type of lists over $B$, with constructors nil, cons, and equip it with the standard snoc operation Path $\times B \rightarrow$ Path. The universal type of infinite trees is

$$
\text { PTree }=\text { Path } \rightarrow \text { ? A }
$$


(where it is crucial that we omit the depth component present in the universal type DTree for initial datatypes). For $f:$ PTree and $p:$ Path, the intended reading of $f p=i n_{i} x$ is that position $p$ in the tree behaves according to the $i$-th alternative and outputs $x: A_{i}$. The carrier of the final $F$-coalgebra is then the subtype $C$ of PTree consisting of those $f$ such that

$$
\begin{gathered}
\operatorname{def}(f \text { nil }) \quad \text { and } \\
\operatorname{def}\left(f\left(\operatorname{snoc} p\left(i n_{i} y\right)\right)\right) \Leftrightarrow \exists x: A_{i} \bullet f p=i n_{i} x
\end{gathered}
$$

for all $i=1, \ldots, n$. (Note that $f p=i n_{i} x$ entails that $f p$ is defined.) We then define an $F$-coalgebra structure $c: C \rightarrow \sum_{i=1}^{n}\left(A_{i} \times\left(B_{i} \rightarrow C\right)\right)$, with injections again denoted $i n_{i}$, by

$$
c f=\text { case } f \text { nil of }\left(i_{i} x \rightarrow i n_{i}\left(x, \lambda y: B_{i} \bullet ! \lambda p: \text { Path } \bullet f\left(\text { cons }\left(i n_{i} y\right) p\right)\right)\right)_{i=1, \ldots, n} \text {. }
$$

Given a further $F$-coalgebra $d: D \rightarrow \sum_{i=1}^{n}\left(A_{i} \times\left(B_{i} \rightarrow D\right)\right)$, we define the morphism $u=$ unfold $d: D \rightarrow C$ recursively by

$$
\begin{aligned}
u z \text { nil } & =\text { case } d z \text { of }\left(i n_{i}(x, g) \rightarrow i n_{i} x\right)_{i=1, \ldots, n} \\
u z\left(\text { cons }\left(i n_{i} y\right) p\right) & =\text { case d } z \text { of } i n_{i}(x, g) \rightarrow u(g y) p
\end{aligned}
$$

where omitted cases in the second case statement are understood to be undefined - we are lucky enough to have the undefined term $(u z p) \uparrow \perp$ available for this purpose (see Remark 5.5). Since primitive recursion on lists is given by an operator, the above definition can be expressed as a term defining unfold $d$ and indeed unfold as a function. It is immediate that $u z$ satisfies (5.1); one proves by induction over $p$ that $u z$ satisfies also (5.2) and therefore indeed belongs to $C$. One verifies directly that $u$ satisfies the defining equation for unfold $d$. Finally, one shows by induction over Path that $u$ is uniquely determined by the unfold equation.

Remark 5.11. The proof of Theorem 5.9 (b) can be modified to prove that quasitoposes have so-called $M$-types [34]. We have omitted this aspect from the main line of the presentation, as it involves the use of dependent types (which exist in pccc's with equality [25]) and is not relevant for the semantics of HASCASL. We sketch some details for the interested reader, who may note that, discounting the need for the extra machinery of dependent types, the formulation of the proof is in fact slightly simpler in the case of $M$-types.

An $M$-type is defined as a final coalgebra for general polynomial functors, i.e. functors $P_{q}$ defined by

$$
P_{q}(X)=\sum a: A \cdot\left(q^{-1}(a) \rightarrow X\right)
$$

where $q: B \rightarrow A$ is a morphism, thought of as a dependent type $\left(B_{a}\right)_{a: A}$ with $B_{a}=q^{-1}(a)$, and the sum is a dependent sum, consisting of pairs $(a, h)$ with $a: A$ and $h: B_{a} \rightarrow X$; one has projections $\pi_{1}, \pi_{2}$ with $\pi_{1}(a, h)=a$ and $\pi_{2}(a, h)=h$. One lets these $A, B$ play the roles of $A, B$, respectively, as in the proof of Theorem 5.9, then Equation 5.2 in the definition of $C$ becomes

$$
\operatorname{def}(f(\operatorname{snoc} p b)) \Leftrightarrow q b=f p .
$$

The definition of the $P_{q}$-coalgebra structure on $C$ is now

$$
c f=\left(f n i l, \lambda b: B_{f n i l} \bullet ! \lambda p: \text { Path } \bullet f(\text { cons } b p)\right) \text {. }
$$


Finally, the unique coalgebra morphism from $u=$ unfold $d: D \rightarrow C$ from a further $P_{q}$-coalgebra $d: D \rightarrow P_{q}(D)$ into $(C, c)$ is recursively defined by

$$
\begin{aligned}
u z \text { nil } & =\pi_{1}(d z) \\
u z(\text { cons } b p) & =u\left(\pi_{2}(d z) b\right) p
\end{aligned}
$$

on the understanding that $B_{a}$ is a subtype of $B$ and that functions $B_{a} \rightarrow X$ (such as $\left.\pi_{2}(d z): B_{\pi_{1}(d z)} \rightarrow X\right)$ extend to partial functions $B \rightarrow ? X$ defined precisely on $B_{a}$ (this is in agreement with the coding of dependent types in pccc's with equality according to [25]). The proof thus modified establishes that pccc's with equality, finite coproducts, and nno, in particular quasitoposes with nno, have $M$-types.

The diligent reader may wonder where the minor trick went that we had to apply in the construction of $u$ in the proof of Theorem 5.9 (b): recall that we needed an undefined term of type $A$, which we obtained as $(u z p) \uparrow \perp$. No such thing is needed above (although, of course, the term is still available). The answer to this puzzle is that unless coproducts are disjoint, which by Lemma 5.3 gives us a constant bot $: 1 \rightarrow$ ? a at every type $a, M$-types do not generalise the final coalgebras of Theorem 5.9, since general polynomial functors do not actually generalise extended polynomial functors in the sense of the theorem. As a simple example, let $A=1+1$ and $B=B_{l}+B_{r}$, with the obvious projection $q: B \rightarrow A$. Theorem 5.9 yields a final coalgebra for the extended polynomial functor $F(X)=\left(B_{l} \rightarrow\right.$ $X)+\left(B_{r} \rightarrow X\right)$, while the $M$-type considered above is a final coalgebra for the functor $P_{q}(X)=\sum a: A \cdot\left(q^{-1}(a) \rightarrow X\right)$. Although one tends to believe that the two functors should be isomorphic, this is not in general true unless coproducts are disjoint. To see this, consider the construction of $\sum a: A \cdot\left(q^{-1}(a) \rightarrow X\right)$ according to [25], which is just a reformulation of the natural set-theoretic description:

$$
P_{q}(X)=(f: B \rightarrow ? X ; a: A . \forall b: B . \operatorname{def}(f b) \Leftrightarrow q b=a) .
$$

Using bot, we can construct an isomorphism $h: F(X) \rightarrow P_{q}(X)$ by

$$
h(\text { inl } f)=((\lambda b: B \text { • case b of inl } x \rightarrow f x \mid \text { inr } y \rightarrow \text { bot }()) \text {, inl }()),
$$

analogously on the other summand, but without bot, the construction of $h$ is not possible. Indeed, a simple example shows that $P_{q}$ and $F$ need not be isomorphic in general. Recall the category $\operatorname{Spa}(\mathcal{P})$ from Remark 5.4, which has non-disjoint coproducts. Let $B_{l}$ be the object $1=(\{*\}, \mathcal{P}(\{*\}))$ of this category, and let $B_{r}$ be the object $1_{\emptyset}=(\{*\}, \emptyset)$. Then $F$ as above has $F\left(1_{\emptyset}\right)=\left(1 \rightarrow 1_{\emptyset}\right)+\left(1_{\emptyset} \rightarrow 1_{\emptyset}\right) \neq \emptyset$ (the right hand summand contains the identity map). However, $P_{q}\left(1_{\emptyset}\right)=\emptyset$, as there is no partial morphism $f: B=B_{l}+B_{r}=1+1_{\emptyset} \rightarrow$ ? $1_{\emptyset}$, because the structure of $1+1_{\emptyset}$ contains the empty subset.

Both part (b) of Theorem 5.9 and the above existence proof for $M$-types in quasitoposes complement recent results of van den Berg and De Marchi [34] (extending earlier work by Santocanale 24]), which live in the setting of locally cartesian closed categories with disjoint coproducts and nno. In particular, existence of $M$-types in quasitoposes with disjoint coproducts, while not formally stated in [34, would seem to follow by a straightforward adaptation of the arguments used there (specifically, exchange decidable subobjects for regular subobjects in the proof of Proposition 4.4 in [34]). We point out that in our setting, we obtain a comparatively simple construction of $M$-types - in the presence of partial function types, one can write down the $M$-type directly as a type of certain partial functions on paths, while the framework of [34 requires a more roundabout approach involving in particular the construction of infinite trees as sequences of finite-depth approximations. 
Remark 5.12. The crucial difference between the above proof and the constructions of 18 , [2], which are also the basis of the topos-theoretic arguments in [10], is the definition of the universal types as partial function spaces rather than types of sets of nodes, reflecting the fact that functional relations need not be functions in the absence of unique choice. Moreover, the construction of primitive recursive functions can no longer rely on an inductive construction of their graphs. It is an open problem whether our use of the depth function for this purpose in the case of initial datatypes can be generalised so as to cover also infinitely branching datatypes such as the type Tree $a b$ from Example 2.2, or more generally $W$ types, i.e. initial algebras for general polynomial functors $P_{q}$ as in Remark 5.11 (such types do exist in toposes with nno [16, 10]; even more generally, the existence of $W$-types implies the existence of initial algebras for dependent polynomial functors [5]).

\section{Domains}

The treatment of general recursion in HASCASL is based on a HOLCF-style [21] internal representation of domains, phrased in terms of chain-complete partial orders. Some adaptations to this theory are necessary in order to cope with the absence of unique choice [28]. We briefly recall the relevant definitions and results below, and then go on to discuss the existence of initial datatypes in the category of domains. As already in the case of datatypes, we work in the internal language of a pccc with equality, sums, and nno; additionally, we assume disjointness of coproducts.

The main difficulty is that without unique choice, we can no longer e.g. define the value at $x$ of the supremum of a chain of partial functions $f_{i}$ as 'the value (if any) eventually assumed by the $f_{i}(x)^{\prime}$. Hence the modified definition

Definition 6.1. A partial order $A$ with ordering $\sqsubseteq$ is called a complete partial order (cpo) if the type $1 \rightarrow$ ? A, equipped with the ordering

$$
x \sqsubseteq y \Leftrightarrow(\operatorname{def} x() \Rightarrow x() \sqsubseteq y()),
$$

has suprema of chains, denoted by $\bigsqcup$, and a bottom element $\perp$ (the latter is not, of course, a bottom element of $A$ itself). We call chains in $1 \rightarrow$ ? A partial chains. We say that a cpo $A$ is pointed (or a cppo) if $A$ has a bottom element. We say that $A$ is a flat cpo if $A$ is a cpo when equipped with the discrete ordering. A partial function between cpo's is continuous iff it preserves suprema of partial chains. The types of total and partial continuous functions from $A$ to $B$ are denoted $A \stackrel{c}{\longrightarrow} B$ and $A \stackrel{c}{\longrightarrow}$ ? $B$, respectively.

Lemma 6.2. Let $\left(x_{i}\right)$ be a partial chain. Then $\bigsqcup_{i} x_{i}$ is defined iff $\exists n \bullet$ def $x_{n}$.

Cpo's can be specified as a class in HASCASL; this is carried out in detail in [28. While under unique choice, all types can be made into flat cpo's, this need not be the case without unique choice. Cppo's in the above sense have least fixed points of continuous endofunctions $f$, constructed as suprema of (total) chains $\left(f^{n} \perp\right)$; this is the basis of the interpretation of general recursive functions. Cpo's are closed under the usual type constructors:

Proposition 6.3. Let $A$ and $B$ be cpo's. Then $A \times B$, equipped with the componentwise ordering, is a cpo.

Proposition 6.4. Let $A$ and $B$ be cpo's, and let $C$ be a type. Then the types $C \rightarrow B$, $C \rightarrow ? B, A \stackrel{c}{\longrightarrow} B$, and $A \stackrel{c}{\longrightarrow}$ ? B are cpo's when equipped with the componentwise ordering; $C \rightarrow ? B$ and $A \stackrel{c}{\longrightarrow}$ ? B are moreover pointed. 
Proposition 6.5. The unit type is a cpo.

Corollary 6.6. If $A$ is a cpo, then $1 \rightarrow$ ? A is a cppo.

In general, the sum of two cpo's, even Bool $=1+1$, need not be a cpo when equipped with the sum ordering. However, we have

Lemma 6.7. Cpo's are stable under sums of partial orders iff Bool is a flat cpo.

Remark 6.8. The previous lemma is the crucial point where disjointness of coproducts (in the shape of bot) is needed. One could alternatively just assume that cpo's are stable under sums of partial orders, but this is conceptually not entirely satisfactory.

The syntactic sugaring of domains in HASCASL includes a free domain construct that declares initial algebras in the category of cpo's and continuous functions (rather than in the category of types and functions as in the case of free type). We now show that the initial datatypes and final process types for polynomial and extended polynomial functors $F$, respectively, constructed in the proof of Theorem 5.9 can be made (respectively, in the case of final process types, slightly modified) into cpo's in such a way that they become initial algebras and final coalgebras, respectively, for the corresponding functor, denoted $\bar{F}$, on the category of cpo's and continuous functions, where in the case of extended polynomial functors, function spaces are replaced by continuous function spaces. It is an important open problem whether this result can be extended to datatypes $t$ with non-strict constructors, i.e. with arguments of type $1 \rightarrow$ ? $t$, such as the type of lazy lists. In the following, we assume that Nat is a flat cpo (this may or may not be the case in concrete models [28]); consequently, Bool is also a flat cpo, and hence cpo's are stable under sums by Lemma 6.7.

Initial Datatypes as Cpo's. Let $T$ be the initial algebra for the functor $F X=\sum_{i=1}^{n}\left(A_{i} \times\right.$ $\left.X^{k_{i}}\right)$ as in Sect. 5, where the parameter objects $A_{i}$ are cpo's. Then the ordering on $T$ is inherited, reusing here and below the notation from the proof of Theorem 5.9, from DTree (this is equivalent to the obvious recursive definition of a componentwise ordering), which by the above results and under the given assumptions is a cppo.

Proposition 6.9. With the above ordering, $T$ is an initial $\bar{F}$-algebra in the category of cpo's and continuous functions.

Proof. It is easy to see that the constructors $c_{i}$ as defined in the proof of Theorem 5.9 are continuous. To prove that $T$ is a cpo, it suffices to show that the supremum in DTree of a partial chain $s$ in $T$ is again in $T$, provided that $\sup s$ is a defined value in DTree. We proceed by induction over depth $\bigsqcup s_{m}$. Let $s_{m}=\left(l_{m}, d_{m}\right)$ for all $m$, and let $\sup s_{m}=(l, d)$. Then $l n i l=i n_{i}$ () for some $i$. By the definition of the sum ordering and Lemma 6.2, there is some $m$ such that $l_{r}$ nil $=i n_{i}$ () for all $r \geq m$. Since $l_{r}$ is in $T$, we have $s_{r}=$ $c_{i}\left(\right.$ leaf $_{i} s_{r}$, sel $_{1} s_{r}, \ldots$, sel $\left._{k_{i}} s_{r}\right)$ and $\operatorname{sel}_{j} s_{r}: T$ for $j=1, \ldots, k_{i}$ and $r \geq m$. By continuity of $c_{i}$, it now follows from the inductive assumption that $\sup s_{m}$ belongs to $T$.

It remains to be shown that for continuous functions $b_{i}$ representing a $\bar{F}$-algebra on a cpo $B$, the function fold $b_{1} \ldots b_{n}: T \rightarrow B$ is continuous. It is easy to see that, given the auxiliary function $f: N a t \rightarrow T \rightarrow$ ? B from the proof of Theorem [5.9, the function $f n$ is continuous for every $n$ in Nat. Since Nat is equipped with the flat ordering, it follows that $f$ itself is continuous. Continuity of fold $b_{1} \ldots b_{n}=\lambda z \bullet f($ depth $z) z$ then follows by the (obvious) continuity of depth. In fact, $f$ even depends continuously on the $b_{i}$, so that fold itself is continuous. 
We have thus established that

the category of cpo's in a pccc with equality, disjoint finite coproducts, and nno has initial algebras of polynomial functors if the nno is a flat cpo,

and hence that declarations of free domains for polynomial functors in HASCASL are conservative as extensions of the specification of sums and a flat cpo of natural numbers; moreover, the above proof shows additionally that the fold operator, and hence the primitive recursion operator, is a continuous higher order function.

Final Process Types as Cpo's. Unlike in the case of initial datatypes, we have to modify the universal type PTree to the type

$$
\text { CPTree }=\text { Path } \stackrel{c}{\longrightarrow} ? A,
$$

again reusing the notation from the proof of Theorem [5.9, in order to obtain a coalgebra structure for $\bar{F}$. By the above results, including the fact that list types are cpo's, CPTree is a cppo. The definition of the subtype $C$, the structure map $c: C \rightarrow \bar{F} C$, and the function $u=$ unfold $d: D \rightarrow C$ for a continuous $\bar{F}$-coalgebra $d$ on a cpo $D$ are otherwise literally the same as in the proof of Theorem 5.9. It is easy to see that $C$ is closed under suprema of chains in CPTree and hence a cpo. Since $C$ consists of continuous maps, $c f$ is really in $\bar{F} C$ (where functions $B_{i} \stackrel{c}{\longrightarrow} C$ must be continuous) for $f: C$. It is straightforward to check that $c$ and $u$ are continuous, using in the latter case the fact established above that primitive recursive functions (here, on Path), as well as the primitive recursion operator itself, are continuous. We have thus shown that

the category of cpo's in a pccc with equality, disjoint finite coproducts, and nno has final coalgebras of extended polynomial functors if the nno is a flat cpo

and hence that corresponding declarations of final process types as cpo's for extended polynomial functors in HASCASL are conservative as extensions of the specification of sums and a flat cpo of natural numbers. (Recall that such declarations are not a HAsCAsL language feature as such, but can be emulated according to Sect. 3 and 4 .)

\section{Conclusion}

We have laid out how initial datatypes and final process types are incorporated into HASCASL, and we have established the existence of such types for a broad class of signature formats. The main contribution in the latter respect is the avoidance of the unique choice principle, which means that, on a more abstract level, our constructions work in any quasitopos (more precisely, in any partial cartesian closed category with equality and finite coproducts) with a natural numbers object. We have moreover discussed how the constructions can be adapted to yield corresponding types with a domain structure as used in HASCASL's internal modelling of general recursion.

We have remarked that our construction of final process types can be modified to prove existence of so-called $M$-types [34, i.e. final coalgebras for general polynomial functors, defined over signatures given in terms of an arbitrary morphisms $q: B \rightarrow A$. While toposes with nno also have $W$-types, i.e. initial algebras for such functors [10, the extension of our construction of initial datatypes beyond finite branching remains an open problem. A further point of interest for future research are datatypes with lazy constructors, such 
as the type of lazy lists, in quasitoposes, and in particular in the category of internal cpo's in a quasitopos. Support for datatypes with finitary polynomial signatures is already implemented in the heterogeneous tool set Hets [12]; support for more complex signatures, intertwined with HASCASL's type class mechanism as described here, is forthcoming.

\section{ACKNOWLEDGEMENTS}

The author wishes to thank Till Mossakowski, Christoph Lüth, Christian Maeder, and Bernd Krieg-Brückner for collaboration on HASCASL, Peter Johnstone and Sam Staton for helpful remarks, and the anonymous referees for their valuable suggestions for improvement of the paper. In particular, the second referee has provided useful hints on $M$-types and disjointness of coproducts. Moreover, Erwin R. Catesbeiana has voiced his opinion on conservative extensions.

\section{REFERENCES}

[1] J. Adámek, H. Herrlich, and G. E. Strecker. Abstract and Concrete Categories. Wiley Interscience, 1990.

[2] S. Berghofer and M. Wenzel. Inductive datatypes in HOL - lessons learned in formal-logic engineering. In Y. Bertot, G. Dowek, A. Hirschowitz, C. Paulin, and L. Théry, eds., Theorem Proving in Higher Order Logics, TPHOLs 1999, vol. 1690 of Lect. Notes Comput. Sci., pp. 19-36. Springer, 1999.

[3] M. Bidoit and P. D. Mosses. CAsl User Manual, vol. 2900 of Lect. Notes Comput. Sci. Springer, 2004.

[4] L. Birkedal and R. E. Møgelberg. Categorical models for Abadi and Plotkin's logic for parametricity. Math. Struct. Comput. Sci., 15, 2005.

[5] N. Gambino and M. Hyland. Wellfounded trees and dependent polynomial functors. In S. Berardi, M. Coppo, and F. Damiani, eds., Types for Proofs and Programs, TYPES 2003, vol. 3085 of Lect. Notes Comput. Sci., pp. 210-225. Springer, 2003.

[6] J.-Y. Girard. Proofs and Types. Cambridge University Press, 1989. Translated and with appendices by P. Taylor and Y. Lafont.

[7] E. L. Gunter. A broader class of trees for recursive type definitions for HOL. In J. J. Joyce and C.-J. H. Seger, eds., Higher Order Logic Theorem Proving and Its Applications, HUG 1993, vol. 780 of Lect. Notes Comput. Sci., pp. 141-154. Springer, 1993.

[8] O. Kiselyov, R. Lämmel, and K. Schupke. Strongly typed heterogeneous collections. In H. Nilsson, ed., Haskell Workshop, Haskell 2004, pp. 96-107. ACM, 2004.

[9] J. Lambek and P. J. Scott. Introduction to Higher-Order Categorical Logic. Cambridge, 1986.

[10] I. Moerdijk and E. Palmgren. Wellfounded trees in categories. Ann. Pure Appl. Logic, 104:189-218, 2000.

[11] E. Moggi. Categories of partial morphisms and the $\lambda_{p}$-calculus. In D. H. Pitt, S. Abramsky, A. Poigné, and D. E. Rydeheard, eds., Category Theory and Computer Programming, vol. 240 of Lect. Notes Comput. Sci., pp. 242-251. Springer, 1986.

[12] T. Mossakowski, C. Maeder, and K. Lüttich. The Heterogeneous Tool Set. In O. Grumberg and M. Huth, eds., Tools and Algorithms for the Construction and Analysis of Systems, TACAS 200\%, vol. 4424 of Lect. Notes Comput. Sci., pp. 519-522. Springer, 2007.

[13] T. Mossakowski, L. Schröder, M. Roggenbach, and H. Reichel. Algebraic-co-algebraic specification in CoCasl. J. Logic Algebraic Programming, 67:146-197, 2006.

[14] P. D. Mosses, ed. CAsL Reference Manual, vol. 2960 of Lect. Notes Comput. Sci. Springer, 2004.

[15] T. Nipkow, L. C. Paulson, and M. Wenzel. Isabelle/HOL - A Proof Assistant for Higher-Order Logic, vol. 2283 of Lect. Notes Comput. Sci. Springer, 2002.

[16] R. Paré and D. Schumacher. Abstract families and the adjoint functor theorems. In P. Johnstone and R. Paré, eds., Indexed categories and their applications, vol. 661 of Lect. Notes Math., pp. 1-125. Springer, 1978.

[17] L. C. Paulson. Set theory for verification. II: Induction and recursion. J. Autom. Reasoning, 15:167-215, 1995. 
[18] L. C. Paulson. Mechanizing coinduction and corecursion in higher-order logic. J. Log. Comput, 7:175204, 1997.

[19] W. Phoa. An introduction to fibrations, topos theory, the effective topos and modest sets. Research report ECS-LFCS-92-208, Lab. for Foundations of Computer Science, University of Edinburgh, 1992.

[20] A. Pitts. Categorical logic. In S. Abramsky, D. Gabbay, and T. Maibaum, eds., Handbook of Logic in Computer Science, vol. 5, Algebraic and Logical Structures, chapter 2. Oxford University Press, 2000.

[21] F. Regensburger. HOLCF: Higher order logic of computable functions. In E. T. Schubert, P. J. Windley, and J. Alves-Foss, eds., Theorem Proving in Higher Order Logics, TPHOLS 1995, vol. 971 of Lect. Notes Comput. Sci., pp. 293-307, 1995.

[22] G. Rosolini. Continuity and Effectiveness in Topoi. PhD thesis, Merton College, Oxford, 1986.

[23] G. Rosolini and T. Streicher. Comparing models of higher type computation. In L. Birkdedal, J. van Oosten, G. Rosolini, and D. S. Scott, eds., Realizability Semantics and Applications, vol. 23 of Electron. Notes Theoret. Comput. Sci., 1999.

[24] L. Santocanale. Logical construction of final coalgebras. In H. P. Gumm, ed., Coalgebraic Methods in Computer Science, CMCS 2003, vol. 82 of Electron. Notes Theoret. Comput. Sci. Elsevier, 2003.

[25] L. Schröder. The logic of the partial $\lambda$-calculus with equality. In J. Marcinkowski and A. Tarlecki, eds., Computer Science Logic, vol. 3210 of Lect. Notes Comput. Sci., pp. 385-399. Springer, 2004.

[26] L. Schröder. The HASCASL prologue - categorical syntax and semantics of the partial $\lambda$-calculus. Theoret. Comput. Sci., 353:1-25, 2006.

[27] L. Schröder. Bootstrapping types and cotypes in HasCasL. In T. Mossakowski and U. Montanari, eds., Algebra and Coalgebra in Computer Science, CALCO 200\%, vol. 4624 of Lect. Notes Comput. Sci., pp. 447-462. Springer, 2007.

[28] L. Schröder and T. Mossakowski. HasCASL: Integrated higher-order specification and program development. Theoret. Comput. Sci. To appear.

[29] L. Schröder and T. Mossakowski. HASCASL: Towards integrated specification and development of Haskell programs. In H. Kirchner and C. Ringeissen, eds., Algebraic Methodology and Software Technology, AMAST 2002, vol. 2422 of Lect. Notes Comput. Sci., pp. 99-116. Springer, 2002.

[30] L. Schröder and T. Mossakowski. Monad-independent Hoare logic in HasCAsL. In M. Pezzè, ed., Fundamental Approaches to Software Engineering, FASE 2003, vol. 2621 of Lect. Notes Comput. Sci., pp. 261-277. Springer, 2003.

[31] L. Schröder and T. Mossakowski. Generic exception handling and the Java monad. In C. Rattray, S. Maharaj, and C. Shankland, eds., Algebraic Methodology and Software Technology, AMAST 2004, vol. 3116 of Lect. Notes Comput. Sci., pp. 443-459. Springer, 2004.

[32] L. Schröder and T. Mossakowski. Monad-independent dynamic logic in HasCasL. J. Logic Comput., 14:571-619, 2004.

[33] L. Schröder, T. Mossakowski, and C. Lüth. Type class polymorphism in an institutional framework. In J. Fiadeiro, ed., Recent Developments in Algebraic Development Techniques, 17th International Workshop, WADT 04, vol. 3423 of Lect. Notes Comput. Sci., pp. 234-248. Springer, 2004.

[34] B. van den Berg and F. De Marchi. Non-well-founded trees in categories. Ann. Pure Appl. Logic, 146:40$59,2007$.

[35] D. Walter, L. Schröder, and T. Mossakowski. Parametrized exceptions. In J. Fiadeiro and J. Rutten, eds., Algebra and Coalgebra in Computer Science, CALCO 05, vol. 3629 of Lect. Notes Comput. Sci., pp. 424-438. Springer, 2005.

[36] O. Wyler. Lecture notes on topoi and quasitopoi. World Scientific, 1991. 